\title{
OPTIMAL CONTRACTS IN CONTINUOUS-TIME MODELS
}

\author{
JAKŠA CVITANIĆ, XUHU WAN, AND JIANFENG ZHANG \\ Received 17 November 2005; Revised 3 February 2006; Accepted 19 February 2006
}

We present a unified approach to solving contracting problems with full information in models driven by Brownian motion. We apply the stochastic maximum principle to give necessary and sufficient conditions for contracts that implement the so-called first-best solution. The optimal contract is proportional to the difference between the underlying process controlled by the agent and a stochastic, state-contingent benchmark. Our methodology covers a number of frameworks considered in the existing literature. The main finance applications of this theory are optimal compensation of company executives and of portfolio managers.

Copyright (C) 2006 Jakša Cvitanić et al. This is an open access article distributed under the Creative Commons Attribution License, which permits unrestricted use, distribution, and reproduction in any medium, provided the original work is properly cited.

\section{Introduction}

In the recent years there has been a significant revival of interest in the so-called risksharing problems and principal-agent problems from economics and finance, especially in continuous time models. Main applications in finance include optimal reward of portfolio managers and optimal compensation of company executives.

The problem involves an interaction between two parties to a contract: an agent and a principal. Through the contract, the principal tries to induce the agent to act in line with the principal's interests. In our setting, both the principal and the agent have full information. In such a case, the problem is known as risk-sharing, since the optimal solution maximizes a combination of the objective functions of the principal and the agent, and represents the best way to share the risk between them. This solution is called the firstbest solution. However, in addition to risk-sharing, we focus on the question of finding as simple as possible contracts which induce the agent to implement actions which will lead to the principal attaining the first-best utility.

In this paper, we consider principal-agent problems in continuous time, in which both the volatility (the diffusion coefficient) and the drift of the underlying process can 
be controlled by the agent. The pioneering paper in the continuous-time framework is Holmström and Milgrom [5], which showed that if both the principal and the agent have exponential utilities, then the optimal contract is linear. Their framework, however, is not that of risk-sharing, but of so-called "hidden information" or "moral hazard" case: the principal cannot directly observe the actions of the agent, who controls the drift only. We consider the (harder) hidden information case in a follow-up paper, by Cvitanić et al. [2], in more general models.

Here, as mentioned above, we study the full information case, in which the principal can observe the agent's actions. Although often less realistic than the hidden information case, we would like to point out that our full information framework is directly applicable to the important finance problem of optimal reward of portfolio managers. We will see that in such a context the optimal contracts do not require observing the manager's actions anyway. We would also like to point out that the extension to continuous-time models is important for these reasons: such a model is appropriate for portfolio management applications, and it also brings the principal-agent literature in line with popular option pricing models. This is especially important now when US companies are required to price employee/executive options.

In the existing literature under full information, it is usually assumed that the principal would force the agent to implement the controls which are best for the principal, the first-best solution. Instead, we study the implementability of the first-best solution by relatively simple contracts, and show that the contract that implements the solution is proportional to the difference between the terminal value of the underlying process and a stochastic, state-contingent benchmark. This should be of significant interest in financial economics, because it justifies the use of linear contracts (paying "shares" rather than "options"), as long as we allow the remaining payment to be a random outcome of a specific benchmark portfolio. It can also be interpreted, in the firm context, as a contract in which the principal sells the firm to the agent in exchange for a specific random payment at a given future time.

Literature on the first-best case in continuous time includes Müller [10, 11], who finds the solution in the exponential utilities case, when the drift is controlled, and shows how it can be approximated by control revisions taking place at discrete times. Very general framework with several agents and recursive utilities is considered in Duffie et al. [3] and Dumas et al. [4]. Ou-Yang [13] also considers the principal-agent problem in the context of delegated portfolio management. In his paper, the agent controls the volatility and the drift simultaneously. While he restricts the family of allowable contracts, motivated by the fact that the principal may not observe full information, the restricted solution of his problem turns out to be the same as the solution of our full information problem, and thus the restriction does not really matter. That paper uses Hamilton-JacobiBellman equations as the technical tool. In Cadenillas et al. [1] the results of Ou-Yang [13] have been generalized to a setting where the drift is also controlled by the agent independently of the volatility, and the principal observes it. They use duality-martingale methods, familiar from the portfolio optimization theory. Because of the limitations of that approach, the results of those two papers are obtained in the setting of linear dynamics (although the cost function is allowed to be nonlinear). Larsen [7] solves numerically 
the case with power utilities for the linear, portfolio delegation case, for contracts which depend only on the final value of the portfolio.

While this and other existing literature usually search for optimal contracts in a specific, reduced family of contracts, we consider completely general contracts, with general diffusion dynamics for the underlying process, and we have general utility functions (separable or not separable) and a general cost function. However, as mentioned above, our contracts often turn out to be of simple form allowed by usual restricted families. We are able to deal with such a general framework by using the "stochastic maximum principle" method of stochastic control theory. (For other applications of stochastic maximum principle in finance, see the recent book by Oksendal and Sulem [12].) In general, it is more straightforward to find explicit solutions (when they exist) from the characterization we obtain, compared to the above-mentioned methods.

We do not discuss the existence of the optimal control. Instead, the stochastic maximum principle enables us to characterize the optimal contract via a solution to forwardbackward stochastic differential equations (FBSDEs), possibly fully coupled. For some of these there is a theory that guarantees existence. However, in general, it is not known under which general conditions these equations have a solution. Nevertheless, we can find optimal contracts in many examples. The stochastic maximum principle is covered in the book by Yong and Zhou [17], while FBSDEs are studied in, for example, [6, 14], and in the monograph by Ma and Yong [9] which contains more references.

The paper is organized as follows: in Section 2 we set up the contracting problem with full information and we find the first-best solution, the one that corresponds to the best controls from the principal's point of view. In Section 3 we show that those controls are implementable, that is, there is a contract which induces the agent to implement the firstbest controls. We present some examples in Section 4. We conclude in Section 5, mentioning possible further research topics.

\section{The first-best solution}

Because we assume that the principal can observe the actions of the agent, it turns out that the principal can induce the agent to use the controls which are optimal for the principal, called the first-best solution. We find the first-best controls in this section. The contract that achieves this solution is called the first-best contract, and we study it in the subsequent section.

2.1. The model. Let $\left\{W_{t}\right\}_{t \geq 0}$ be a $d$-dimensional Brownian motion on a probability space $(\Omega, \mathscr{F}, P)$, and denote by $\mathbf{F}:=\left\{\mathscr{F}_{t}\right\}_{t \leq T}$ its augmented filtration on the interval $[0, T]$. The controlled state process is denoted $X=X^{u, v}$ and its dynamics are given by

$$
d X_{t}=f\left(t, X_{t}, u_{t}, v_{t}\right) d t+v_{t} d W_{t}
$$

where $(X, u, v)$ take values in $\mathbb{R} \times \mathbb{R}^{m} \times \mathbb{R}^{d}$, and $f$ is a function taking values in $\mathbb{R}$, possibly random and such that, as a process, it is F-adapted. The notation $x y$ for two vectors $x, y \in \mathbb{R}^{d}$ indicates the inner product. 
In the principal-agent problems, the principal gives the agent compensation $C_{T}=$ $F(\omega, X)$ at time $T$, where $F: \Omega \times C[0, T] \rightarrow \mathbb{R}$ is a (deterministic) mapping such that $C_{T}$ is $\mathscr{F}_{T}$ measurable. We say that $F$ is a contract. One of the main results of the paper is that the optimal contract, for the problem below, will be of the form $F(\omega, X)=X_{T}-B_{T}(\omega)$ for some $\mathscr{F}_{T}$-measurable random variable ("benchmark") $B_{T}$. The agent chooses the controls $u$ and $v$ in order to maximize his utility:

$$
V_{1}(F):=\sup _{u, v} V_{1}(u, v ; F):=\sup _{u, v} E\left[U_{1}\left(F\left(\omega, X^{u, v}\right), G_{T}^{u, v}\right)\right] .
$$

Here, $G_{T}^{u, v}:=\int_{0}^{T} g\left(t, X_{t}, u_{t}, v_{t}\right) d t$ is the accumulated cost of the agent, and with a slight abuse of notation we use notation $V_{1}$ both for the objective function and its maximum.

Remark 2.1. The control $u$ has the interpretation of the level of effort the agent applies to her projects, which only affects the drift, but not the volatility of $X$. Typically, the higher $u$, the higher the value of $X$. On the other hand, the choice of $v$ represents the choice of the volatility of $X$, although it may also have an impact on the expected value of $X$. We interpret the choice of $v$ as a choice of projects, and the choice of $v$ is related to the mean-variance tradeoff in available projects (higher mean means also higher risk, and the other way round). For example, if we think of the agent as an executive of a company, we may want to assume that she can increase the company value with her effort $u$, without increasing the volatility risk. On the other hand, in the portfolio management application mentioned below, the manager can only affect the portfolio value by simultaneously affecting the drift and the volatility through the choice of $v$. We could be more general and instead of $v$ have the diffusion term equal to $\sigma\left(t, X_{t}, v_{t}\right)$. However, it is often possible to do the transformation of variables $\widetilde{v}_{t} \triangleq \sigma\left(t, X_{t}, v_{t}\right)$, which reduces to the model we study. In particular, in the important application of portfolio management, we can usually take $\sigma\left(t, X_{t}, v_{t}\right)=\sigma_{t} v_{t}$ for some given process $\sigma_{t}$ of invertible matrices, so that the transformation of variables is straightforward. The case of "incomplete markets," in which $\sigma\left(t, X_{t}, v_{t}\right)$ does not cover the same range as $v$, is left for future research.

We say a contract $F$ is implementable if there exists a pair $\left(u^{F}, v^{F}\right)$ such that

$$
V_{1}\left(u^{F}, v^{F} ; F\right)=V_{1}(F)
$$

The principal maximizes her utility:

$$
V_{2}:=\max _{F} E\left[U_{2}\left(X_{T}^{u^{F}, v^{F}}-F\left(\omega, X^{u^{F}, v^{F}}\right)\right)\right]
$$

where the maximum is over all implementable contracts $F$ such that the following participation constraint or individual rationality (IR) constraint holds:

$$
V_{1}(F) \geq R
$$

Functions $U_{1}$ and $U_{2}$ are the utility functions of the agent and the principal. Function $g$ is a penalty function on the agent's effort. Constant $R$ is the reservation utility of the agent and represents the value of the agent's outside opportunities, the minimum value he 
requires to accept the job. The typical cases studied in the literature are the separable utility case, with $U_{1}(x, y)=U_{1}(x)-y$, and the nonseparable case with $U_{1}(x, y)=U_{1}(x-y)$, where, with a slight abuse of notation, we use the same notation $U_{1}$ also for the function of one argument only. We could also have the same generality for $U_{2}$, but this makes less sense from the economics point of view.

We note that it is equivalent to define a contract as a functional $F(u, v, X)$. In the hidden action case, which we study in a follow-up paper, a contract takes the form $F(X)$.

Example 2.2 (the case of portfolio management). A simple motivating example is the model

$$
d X_{t}=\alpha v_{t} d t+v_{t} d W_{t}
$$

with one-dimensional $W$. If we write $v_{t}=\sigma \pi_{t}$ for a constant $\sigma>0$, this model corresponds to a value of a portfolio strategy that invests amount $\pi_{t}$ at time $t$ in a risky stock modeled by a geometric Brownian motion, with volatility $\sigma$, and constant risk-premium $\alpha$. When the cost function $g$ is zero, in a later section we find explicit optimal contracts for this case as an example of our general theory. We also show that in this case we can find a deterministic function $F$ such that the contract $F\left(X_{T}\right)$ is optimal. Moreover, we consider an example from Ou-Yang when the cost function is not zero, but utility functions are exponential.

We now come to the technical part of the problem. Denote by $L_{n}^{2}$ the set of adapted processes $x$ with values in $\mathbb{R}^{n}$ for which $E \int_{0}^{T}\left|x_{t}\right|^{2} d t<\infty$. Also denote by $\partial_{x} U_{1}$ the derivative of $U_{1}$ with respect to the first argument, and with $\partial_{y} U_{1}$ the derivative of $U_{1}$ with respect to the second argument. In this section we impose the following assumptions.

(A1) Functions $f, g:[0, T] \times \mathbb{R} \times \mathbb{R}^{m} \times \mathbb{R}^{d} \times \Omega \rightarrow \mathbb{R}$ are continuously differentiable with respect to $x, u, v$ such that $f_{x}, g_{x}$ are uniformly bounded, and $f_{u}, f_{v}, g_{u}, g_{v}$ have uniform linear growth in $x, u, v$. In addition, $f$ is jointly concave and $g$ is jointly convex in $(x, u, v)$.

(A2) Functions $U_{1}: \mathbb{R}^{2} \rightarrow \mathbb{R}, U_{2}: \mathbb{R} \rightarrow \mathbb{R}$ are differentiable, with $\partial_{x} U_{1}>0, \partial_{y} U_{1}<0$, $U_{2}^{\prime}>0, U_{1}$ is jointly concave, and $U_{2}$ is concave.

(A3) The admissible set $\mathscr{A}$ is the set of all those control triples $\left(C_{T}, u, v\right)$ such that

(i) $u \in L_{m}^{2}, v \in L_{d}^{2}, E\left\{\int_{0}^{T} f\left(t, 0, u_{t}, v_{t}\right)^{2} d t\right\}<\infty, C_{T}$ is an $\mathscr{F}_{T}$-measurable random variable;

(ii) for any bounded $\left(\Delta C_{T}, \Delta u, \Delta v\right)$ satisfying (i), there exists $\varepsilon_{0}>0$ such that

$$
E\left\{\left|U_{1}\left(C_{T}^{\varepsilon}, G_{T}^{\varepsilon}\right)\right|+\left|U_{2}\left(X_{T}^{\varepsilon}-C_{T}^{\varepsilon}\right)\right|\right\}<\infty \quad \forall \varepsilon \in\left[0, \varepsilon_{0}\right],
$$

and $\left|U_{2}^{\prime}\left(X_{T}^{\varepsilon}-C_{T}^{\varepsilon}\right)\right|^{2},\left|\partial_{x} U_{1}\left(C_{T}^{\varepsilon}, G_{T}^{\varepsilon}\right)\right|,\left|\partial_{y} U_{1}\left(C_{T}^{\varepsilon}, G_{T}^{\varepsilon}\right)\right|^{2}$ are uniformly integrable for $\varepsilon \in\left[0, \varepsilon_{0}\right]$, where

$$
\begin{gathered}
C_{T}^{\varepsilon} \triangleq C_{T}+\varepsilon \Delta C_{T} ; \quad u_{t}^{\varepsilon} \triangleq u_{t}+\varepsilon \Delta u_{t} ; \quad v_{t}^{\varepsilon} \triangleq v_{t}+\varepsilon \Delta v_{t} ; \\
X_{t}^{\varepsilon}=x+\int_{0}^{t} f\left(s, X_{s}^{\varepsilon}, u_{s}^{\varepsilon}, v_{s}^{\varepsilon}\right) d s+\int_{0}^{t} v_{s}^{\varepsilon} d W_{s} ; \quad G_{T}^{\varepsilon} \triangleq \int_{0}^{T} g\left(t, X_{t}^{\varepsilon}, u_{t}^{\varepsilon}, v_{t}^{\varepsilon}\right) d t .
\end{gathered}
$$

(A4) There exists $\left(C_{T}, u, v\right) \in \mathscr{A}$ such that $E\left\{U_{1}\left(C_{T}, G_{T}\right)\right\} \geq R$. 
Remark 2.3. Our method also applies to the case in which $u, v$ are constrained to take values in a convex domain, and/or in which the functions $U_{i}$ may only be defined on convex domains, such as power utilities. In this case, we would need to change the definitions of $\Delta C_{T}, \Delta u, \Delta v$. For example, we would define $\Delta u=\tilde{u}-u$, where $\tilde{u}$ is any other drift control satisfying (A3)(i), such that $\Delta u$ is bounded. Moreover, for our maximum principle conditions (2.47) to hold as equalities, we would need to assume that the optimal triple $\left(\hat{C}_{T}, \hat{u}, \hat{v}\right)$ takes values in the interior of its domain.

Remark 2.4. We will see that our sufficient conditions are valid for a wider set of admissible triples, with $\varepsilon_{0}=0$ in (A3).

Remark 2.5. If $U_{1}, U_{2}$ have polynomial growth, the uniform integrability in (A3)(ii) automatically holds true. If they have exponential growth, there are some discussions on the integrability of exponential processes in Yong [16].

Note that (2.1) has a unique strong solution: by (A3)(i) we have

$$
E\left\{\int_{0}^{T}\left[v_{t}^{2}+f\left(t, 0, u_{t}, v_{t}\right)^{2}\right] d t\right\}<\infty .
$$

Then by boundedness of $\left|f_{x}\right|$ and by standard arguments we get $E\left\{\sup _{t}\left|X_{t}\right|^{2}\right\}<\infty$. We also note that, for $(u, v)$ satisfying $(\mathrm{A} 3)(\mathrm{i})$ and $(\Delta u, \Delta v)$ bounded, $\left(u^{\varepsilon}, v^{\varepsilon}\right)$ also satisfies (A3)(i). In fact, since $f_{u}$ and $f_{v}$ have uniform linear growth, we have

$$
\begin{aligned}
\left|f\left(t, 0, u_{t}^{\varepsilon}, v_{t}^{\varepsilon}\right)-f\left(t, 0, u_{t}, v_{t}\right)\right| & =\left|\int_{0}^{\varepsilon}\left[f_{u}\left(t, 0, u_{t}^{\theta}, v_{t}^{\theta}\right) \Delta u_{t}+f_{v}\left(t, 0, u_{t}^{\theta}, v_{t}^{\theta}\right) \Delta v_{t}\right] d \theta\right| \\
& \leq C\left[1+\left|u_{t}\right|+\left|v_{t}\right|\right] .
\end{aligned}
$$

Then

$$
E\left\{\int_{0}^{T} f\left(t, 0, u_{t}^{\varepsilon}, v_{t}^{\varepsilon}\right)^{2} d t\right\} \leq C E\left\{\int_{0}^{T}\left[f\left(t, 0, u_{t}, v_{t}\right)^{2}+1+\left|u_{t}\right|^{2}+\left|v_{t}\right|^{2}\right] d t\right\}<\infty .
$$

Thus (2.8) also has a unique strong solution.

We first consider the so-called first-best solution: in this setting it is actually the principal who chooses the controls $u$ and $v$, and provides the agent with compensation $C_{T}$ so that the IR constraint is satisfied. In other words, the principal's value function is

$$
\bar{V}_{2}:=\sup _{C_{T}, u, v} E\left[U_{2}\left(X_{T}-C_{T}\right)\right]
$$

where $\left(C_{T}, u, v\right) \in \mathscr{A}$ is chosen so that

$$
E\left[U_{1}\left(C_{T}, G_{T}^{u, v}\right)\right] \geq R
$$

In fact, in Section 3 we will prove that (under some conditions), if ( $\left.\hat{C}_{T}, \hat{u}, \hat{v}\right)$ is the optimal 
solution to this problem, then there is an implementable contract $F$ such that $u^{F}=\hat{u}$, $v^{F}=\hat{v}$, and $F\left(\omega, X^{\hat{u}, \hat{v}}\right)=\widehat{C}$. Then obviously

$$
V_{2}=\bar{V}_{2}
$$

To simplify the notations, from now on we abuse the notation and use $V_{2}$ to denote the right-hand side of (2.12). Also for simplicity, henceforth we use the notation for the case when all the processes are one-dimensional.

In order to solve the optimization problem (2.12), we define the Lagrangian as follows for a given constant $\lambda>0$ :

$$
J\left(C_{T}, u, v ; \lambda\right)=E\left[U_{2}\left(X_{T}^{u, v}-C_{T}\right)+\lambda\left(U_{1}\left(C_{T}, G_{T}^{u, v}\right)-R\right)\right] .
$$

Because of our assumptions, by the standard optimization theory (see Luenberger [8]), we have

$$
V_{2}=\sup _{C_{T}, u, v} J\left(C_{T}, u, v ; \hat{\lambda}\right)
$$

for some $\hat{\lambda}>0$. Moreover, if the maximum is attained in (2.12) by $\left(\hat{C}_{T}, \hat{u}, \hat{v}\right)$, then it is attained by the same triple in the right-hand side of (2.16), and we have

$$
E\left[U_{1}\left(\widehat{C}_{T}, G_{T}^{\hat{u}, \hat{v}}\right)\right]=R
$$

Conversely, if there exists $\hat{\lambda}>0$ and $\left(\hat{C}_{T}, \hat{u}, \hat{v}\right)$ such that the maximum is attained in the right-hand side of (2.16) and such that $E\left[U_{1}\left(\widehat{C}_{T}, G_{T}^{\hat{u}, \hat{v}}\right)\right]=R$, then $\left(\widehat{C}_{T}, \hat{u}, \hat{v}\right)$ is also optimal for the problem $V_{2}$ of (2.12).

2.2. Necessary conditions for optimality. We cannot directly apply standard approaches to deriving necessary conditions for optimality, as presented, for example, in the book Yong and Zhou [17], because our optimization problem has a nonstandard form. Thus, we present here a proof starting from the scratch.

Fix $\lambda$ and suppose that $\left(C_{T}, u, v\right) \in \mathscr{A}$ and $\left(\Delta C_{T}, \Delta u, \Delta v\right)$ is uniformly bounded. Let $\varepsilon_{0}>0$ be the constant determined in (A3)(iii). For $\varepsilon \in\left(0, \varepsilon_{0}\right)$, recall (2.8) and denote

$$
\begin{gathered}
J^{\varepsilon} \triangleq J\left(C_{T}^{\varepsilon}, u^{\varepsilon}, v^{\varepsilon}\right) ; \quad J \triangleq J\left(C_{T}, u, v\right) ; \\
\nabla X_{t}^{\varepsilon} \triangleq \frac{X_{t}^{\varepsilon}-X_{t}}{\varepsilon} ; \quad \nabla G_{T}^{\varepsilon} \triangleq \frac{G_{T}^{\varepsilon}-G_{T}}{\varepsilon} ; \quad \nabla J^{\varepsilon} \triangleq \frac{J^{\varepsilon}-J}{\varepsilon} .
\end{gathered}
$$

Moreover, let $\nabla X$ be the solution to the SDE

$$
\nabla X_{t}=\int_{0}^{t}\left[f_{x}(s) \nabla X_{s}+f_{u}(s) \Delta u_{s}+f_{v}(s) \Delta v_{s}\right] d s+\int_{0}^{t} \Delta v_{s} d W_{s}
$$

where $f_{u}(t) \Delta u_{t} \triangleq \sum_{i=1}^{m} \partial_{u_{i}} f\left(t, X_{t}, u_{t}, v_{t}\right) \Delta u_{t}^{i}$, and $f_{x}(t) \nabla X_{t}, f_{v}(t) \Delta v_{t}$ are defined in a similar 
way. By (A1) and (A3)(i) one can easily show that

$$
E\left\{\int_{0}^{T}\left[\left|f_{u} \Delta u_{t}\right|^{2}+\left|f_{v} \Delta v_{t}\right|^{2}+\left|\Delta v_{t}\right|^{2}\right] d t\right\}<\infty .
$$

Thus (2.19) has a strong solution $\nabla X_{t}$ such that $E\left\{\sup _{0 \leq t \leq T}\left|\nabla X_{t}\right|^{2}\right\}<\infty$.

The following lemmas show that the finite difference quotients in (2.18) converge.

Lemma 2.6. Assume (A1) and (A3)(i). Then $\lim _{\epsilon \rightarrow 0} E\left\{\sup _{0 \leq t \leq T}\left|\nabla X_{t}^{\varepsilon}-\nabla X_{t}\right|^{2}\right\}=0$.

Proof. First, by standard arguments one can easily show that

$$
\lim _{\epsilon \rightarrow 0} E\left\{\sup _{0 \leq t \leq T}\left|X_{t}^{\varepsilon}-X_{t}\right|^{2}\right\}=0
$$

Next, we note that

$$
\nabla X_{t}^{\varepsilon}=\int_{0}^{t}\left[\alpha_{s}^{\varepsilon} \nabla X_{s}^{\varepsilon}+\beta_{s}^{\varepsilon} \Delta u_{s}+\gamma_{s}^{\varepsilon} \Delta v_{s}\right] d s+\int_{0}^{t} \Delta v_{s} d W_{s}
$$

where

$$
\begin{gathered}
\alpha_{t}^{\varepsilon} \triangleq \int_{0}^{1} f_{x}\left(t, X_{t}+\theta\left(X_{t}^{\varepsilon}-X_{t}\right), u_{t}^{\varepsilon}, v_{t}^{\varepsilon}\right) d \theta \\
\beta_{t}^{\varepsilon} \triangleq \int_{0}^{1} f_{u}\left(t, X_{t}, u_{t}^{\theta \varepsilon}, v_{t}^{\theta \varepsilon}\right) d \theta ; \quad \gamma_{t}^{\varepsilon} \triangleq \int_{0}^{1} f_{v}\left(t, X_{t}, u_{t}^{\theta \varepsilon}, v_{t}^{\theta \varepsilon}\right) d \theta .
\end{gathered}
$$

By (A1) and the fact that $\Delta u, \Delta v$ are bounded, there exists a constant $C>0$ independent of $\varepsilon$ such that

$$
\left|\alpha_{t}^{\varepsilon}\right| \leq C, \quad\left|\beta_{t}^{\varepsilon}\right|+\left|\gamma_{t}^{\varepsilon}\right| \leq C\left[1+\left|X_{t}\right|+\left|u_{t}\right|+\left|v_{t}\right|\right]
$$

Denote $\Delta \nabla X^{\varepsilon} \triangleq \nabla X^{\varepsilon}-\nabla X$ and

$$
\alpha_{t}^{0} \triangleq f_{x}\left(t, X_{t}, u_{t}, v_{t}\right), \quad \beta_{t}^{0} \triangleq f_{u}\left(t, X_{t}, u_{t}, v_{t}\right), \quad \gamma_{t}^{0} \triangleq f_{v}\left(t, X_{t}, u_{t}, v_{t}\right) .
$$

Then

$$
\Delta \nabla X_{t}^{\varepsilon}=\int_{0}^{t}\left[\alpha_{s}^{\varepsilon} \Delta \nabla X_{s}^{\varepsilon}+\left(\alpha_{s}^{\varepsilon}-\alpha_{s}^{0}\right) \nabla X_{s}+\left(\beta_{s}^{\varepsilon}-\beta_{s}^{0}\right) \Delta u_{s}+\left(\gamma_{s}^{\varepsilon}-\gamma_{s}^{0}\right) \Delta v_{s}\right] d s
$$

Denote $\Lambda_{t}^{\varepsilon} \triangleq \exp \left(-\int_{0}^{t} \alpha_{s}^{\varepsilon} d s\right)$. Then

$$
\Delta \nabla X_{t}^{\varepsilon}=\left[\Lambda_{t}^{\varepsilon}\right]^{-1} \int_{0}^{t} \Lambda_{s}^{\varepsilon}\left[\left(\alpha_{s}^{\varepsilon}-\alpha_{s}^{0}\right) \nabla X_{s}+\left(\beta_{s}^{\varepsilon}-\beta_{s}^{0}\right) \Delta u_{s}+\left(\gamma_{s}^{\varepsilon}-\gamma_{s}^{0}\right) \Delta v_{s}\right] d s .
$$


Jakša Cvitanić et al. 9

Since $\left|\Lambda_{t}^{\varepsilon}\right|+\left|\Lambda_{t}^{\varepsilon}\right|^{-1} \leq C$, and $\Delta u, \Delta v$ are bounded, we have

$$
\left|\Delta \nabla X_{t}^{\varepsilon}\right| \leq C \int_{0}^{t}\left[\left|\alpha_{s}^{\varepsilon}-\alpha_{s}^{0}\right|\left|\nabla X_{s}\right|+\left|\beta_{s}^{\varepsilon}-\beta_{s}^{0}\right|+\left|\gamma_{s}^{\varepsilon}-\gamma_{s}^{0}\right|\right] d s .
$$

Recall (2.21) and that $f_{x}, f_{u}, f_{v}$ are continuous. Thus, by (2.24) and the dominated convergence theorem we get $E\left\{\sup _{t}\left|\nabla X_{t}^{\varepsilon}-\nabla X_{t}\right|^{2}\right\} \rightarrow 0$.

Corollary 2.7. Assume (A1)-(A3). Then

$$
\lim _{\epsilon \rightarrow 0} E\left\{\sup _{0 \leq t \leq T}\left|\nabla G_{t}^{\varepsilon}-\nabla G_{t}\right|^{2}\right\}=0 ; \quad \lim _{\epsilon \rightarrow 0} \nabla J^{\varepsilon}=\nabla J,
$$

where

$$
\begin{gathered}
\nabla G_{t} \triangleq \int_{0}^{t}\left[g_{x} \nabla X_{s}+g_{u} \Delta u_{s}+g_{v} \Delta v_{s}\right] d s ; \\
\nabla J \triangleq E\left\{U_{2}^{\prime}\left(X_{T}-C_{T}\right)\left[\nabla X_{T}-\Delta C_{T}\right]+\lambda \partial_{x} U_{1}\left(C_{T}, G_{T}\right) \Delta C_{T}+\lambda \partial_{y} U_{1}\left(C_{T}, G_{T}\right) \nabla G_{T}\right\} .
\end{gathered}
$$

Proof. First, by (A1) and (A3)(i) one can easily show that $E\left\{\sup _{0 \leq t \leq T}\left|\nabla G_{T}\right|^{2}\right\}<\infty$. Similar to the proof of Lemma 2.6 one can prove

$$
\lim _{\epsilon \rightarrow 0} E\left\{\sup _{0 \leq t \leq T}\left|\nabla G_{t}^{\varepsilon}-\nabla G_{t}\right|^{2}\right\}=0 .
$$

We next prove the convergence of $\nabla J^{\varepsilon}$. By (A3)(ii) we know that $\nabla J$ is well defined. Note that

$$
\begin{aligned}
\nabla J^{\varepsilon}=E & \left\{\int_{0}^{1} U_{2}^{\prime}\left(X_{T}-C_{T}+\theta\left[\left(X_{T}^{\varepsilon}-X_{T}\right)-\varepsilon \Delta C_{T}\right]\right) d \theta\left[\nabla X_{T}^{\varepsilon}-\Delta C_{T}\right]\right. \\
& \left.+\lambda \int_{0}^{1} \partial_{x} U_{1}\left(C_{T}+\theta \varepsilon \Delta C_{T}, G_{T}^{\varepsilon}\right) d \theta \Delta C_{T}+\lambda \int_{0}^{1} \partial_{y} U_{1}\left(C_{T}, G_{T}+\theta\left(G_{T}^{\varepsilon}-G_{T}\right)\right) d \theta \nabla G_{T}^{\varepsilon}\right\} .
\end{aligned}
$$

It is then straightforward to verify that the random variable $V^{\varepsilon}-V$ inside the expectation in $\nabla J^{\varepsilon}-\nabla J=: E\left[V^{\varepsilon}-V\right]$ converges to zero almost surely, as $\varepsilon \rightarrow 0$. Thus, we only have to show that $V^{\varepsilon}$, where $\nabla J^{\varepsilon}=E\left[V^{\varepsilon}\right]$, is uniformly integrable. Note that by monotonicity of $U_{2}^{\prime}$ and $\partial_{x} U_{1}, \partial_{y} U_{1}$, we have

$$
\begin{gathered}
\left|\int_{0}^{1} U_{2}^{\prime}\left(X_{T}-C_{T}+\theta\left[\left(X_{T}^{\varepsilon}-X_{T}\right)-\varepsilon \Delta C_{T}\right]\right) d \theta\right| \leq\left|U_{2}^{\prime}\left(X_{T}-C_{T}\right)\right|+\left|U_{2}^{\prime}\left(X_{T}^{\varepsilon}-C_{T}^{\varepsilon}\right)\right| ; \\
|| \int_{0}^{1} \partial_{x} U_{1}\left(C_{T}+\theta \varepsilon \Delta C_{T}, G_{T}^{\varepsilon}\right) d \theta|\leq| \partial_{x} U_{1}\left(C_{T}, G_{T}^{\varepsilon}\right)|+| \partial_{x} U_{1}\left(C_{T}^{\varepsilon}, G_{T}^{\varepsilon}\right) \mid ; \\
\left|\int_{0}^{1} \partial_{y} U_{1}\left(C_{T}, G_{T}+\theta\left(G_{T}^{\varepsilon}-G_{T}\right)\right) d \theta\right| \leq\left|\partial_{y} U_{1}\left(C_{T}, G_{T}\right)\right|+\left|\partial_{y} U_{1}\left(C_{T}, G_{T}^{\varepsilon}\right)\right| .
\end{gathered}
$$


Using this we get, for a generic constant $C$,

$$
\begin{aligned}
E\left[\left|V^{\varepsilon}\right|\right] \leq E\{\mid & U_{2}^{\prime}\left(X_{T}-C_{T}\right)|+| U_{2}^{\prime}\left(X_{T}^{\varepsilon}-C_{T}^{\varepsilon}\right)||\left[\nabla X_{T}^{\varepsilon}-\Delta C_{T}\right] \mid \\
+ & \lambda\left(\left|\partial_{x} U_{1}\left(C_{T}, G_{T}^{\varepsilon}\right)\right|+\left|\partial_{x} U_{1}\left(C_{T}^{\varepsilon}, G_{T}^{\varepsilon}\right)\right|\right)\left|\Delta C_{T}\right| \\
+ & \left.\lambda\left(\left|\partial_{y} U_{1}\left(C_{T}, G_{T}\right)\right|+\left|\partial_{y} U_{1}\left(C_{T}, G_{T}^{\varepsilon}\right)\right|\right)\left|\nabla G_{T}^{\varepsilon}\right|\right\} \\
\leq C E\{\mid & \left.U_{2}^{\prime}\left(X_{T}-C_{T}\right)\right|^{2}+\left|U_{2}^{\prime}\left(X_{T}^{\varepsilon}-C_{T}^{\varepsilon}\right)\right|^{2}+\left|\partial_{x} U_{1}\left(C_{T}, G_{T}^{\varepsilon}\right)\right| \\
& +\left|\partial_{x} U_{1}\left(C_{T}^{\varepsilon}, G_{T}^{\varepsilon}\right)\right|+\left|\partial_{y} U_{1}\left(C_{T}, G_{T}\right)\right|^{2}+\left|\partial_{y} U_{1}\left(C_{T}, G_{T}^{\varepsilon}\right)\right|^{2} \\
& \left.+\left|\left[\nabla X_{T}^{\varepsilon}-\Delta C_{T}\right]\right|^{2}+\left|\nabla G_{T}^{\varepsilon}\right|^{2}\right\} .
\end{aligned}
$$

Note that (A3)(ii) also holds true for variation $(0, \Delta u, \Delta v)$ (maybe with different $\varepsilon_{0}$ ). Recalling Lemma 2.6 and (2.31), we conclude that $V^{\varepsilon}$ are uniformly integrable on $\varepsilon \leq$ $\varepsilon_{0}$, for a small enough $\varepsilon_{0}$, and applying the dominated convergence theorem we get the following $\lim _{\epsilon \rightarrow 0} \nabla J^{\varepsilon}=\nabla J$.

As usual when finding necessary conditions for stochastic control problems, we now introduce appropriate adjoint processes as follows:

$$
\begin{gathered}
Y_{t}^{1}=-\lambda \partial_{y} U_{1}\left(C_{T}, G_{T}\right)-\int_{t}^{T} Z_{s}^{1} d W_{s} \\
Y_{t}^{2}=U_{2}^{\prime}\left(X_{T}-C_{T}\right)-\int_{t}^{T}\left[Y_{s}^{1} g_{x}\left(s, X_{s}, u_{s}, v_{s}\right)-Y_{s}^{2} f_{x}\left(s, X_{s}, u_{s}, v_{s}\right)\right] d s-\int_{t}^{T} Z_{s}^{2} d W_{s} .
\end{gathered}
$$

Each of these is a backward stochastic differential equation (BSDE), whose solution is a pair of adapted processes $\left(Y^{i}, Z^{i}\right), i=1,2$. Note that in the case $U_{1}(x, y)=U_{1}(x)-y$ we have $Y_{t}^{1} \equiv \lambda, Z_{t}^{1} \equiv 0$. Also note that (A3)(ii) guarantees that the solution $\left(Y^{1}, Z^{1}\right)$ to the first BSDE exists. Then by the fact that $f_{x}, g_{x}$ are bounded and by (A3)(ii) again, the solution $\left(Y^{2}, Z^{2}\right)$ to the second BSDE also exists. Moreover, by the BSDE theory, we have

$$
E\left\{\sup _{0 \leq t \leq T}\left[\left|Y_{t}^{1}\right|^{2}+\left|Y_{t}^{2}\right|^{2}\right]+\int_{0}^{T}\left[\left|Z_{t}^{1}\right|^{2}+\left|Z_{t}^{2}\right|^{2}\right] d t\right\}<\infty .
$$

Theorem 2.8. Assume (A1)-(A3). Then

$$
\nabla J=E\left\{\Gamma_{T}^{1} \Delta C_{T}+\int_{0}^{T}\left[\Gamma_{t}^{2} \Delta u_{t}+\Gamma_{t}^{3} \Delta v_{t}\right] d t\right\}
$$

where

$$
\begin{gathered}
\Gamma_{T}^{1} \triangleq \lambda \partial_{x} U_{1}\left(C_{T}, G_{T}\right)-U_{2}^{\prime}\left(X_{T}-C_{T}\right) ; \\
\Gamma_{t}^{2} \triangleq Y_{t}^{2} f_{u}\left(t, X_{t}, u_{t}, v_{t}\right)-Y_{t}^{1} g_{u}\left(t, X_{t}, u_{t}, v_{t}\right) ; \\
\Gamma_{t}^{3} \triangleq Y_{t}^{2} f_{v}\left(t, X_{t}, u_{t}, v_{t}\right)-Y_{t}^{1} g_{v}\left(t, X_{t}, u_{t}, v_{t}\right)+Z_{t}^{2} .
\end{gathered}
$$


Proof. By (2.30) and (2.35), obviously we have

$$
\nabla J=E\left\{\Gamma_{T}^{1} \Delta C_{T}+Y_{T}^{2} \nabla X_{T}-Y_{T}^{1} \nabla G_{T}\right\}
$$

Recalling (2.19), (2.30), and (2.35), and applying Itô's formula we have

$$
d\left(Y_{t}^{2} \nabla X_{t}-Y_{t}^{1} \nabla G_{t}\right)=\left[\Gamma_{t}^{2} \Delta u_{t}+\Gamma_{t}^{3} \Delta v_{t}\right] d t+\Gamma_{t}^{4} d W_{t}
$$

where $\Gamma_{t}^{4} \triangleq Y_{t}^{2} \Delta v_{t}+\nabla X_{t} Z_{t}^{2}-\nabla G_{t} Z_{t}^{1}$. Note that $\nabla X$ and $\nabla G$ are continuous, thus

$$
\sup _{0 \leq t \leq T}\left[\left|\nabla X_{t}\right|^{2}+\left|\nabla G_{t}\right|^{2}\right]<\infty, \quad \text { a.s. }
$$

By (2.36) we have $\int_{0}^{T}\left[\left|Z_{t}^{1}\right|^{2}+\left|Z_{t}^{2}\right|^{2}\right] d t<\infty$, a.s. Therefore,

$$
\int_{0}^{T}\left|\Gamma_{t}^{4}\right|^{2} d t<\infty, \quad \text { a.s. }
$$

Define a sequence of stopping times:

$$
\tau_{n} \triangleq \inf \left\{t>0: \int_{0}^{t}\left|\Gamma_{s}^{4}\right|^{2} d s>n\right\} \wedge T
$$

By (2.42) obviously we have $\tau^{n} \uparrow T$. By (2.40) and noting that $\nabla X_{0}=\nabla G_{0}=0$, we have

$$
E\left\{Y_{\tau_{n}}^{2} \nabla X_{\tau_{n}}-Y_{\tau_{n}}^{1} \nabla G_{\tau_{n}}\right\}=E\left\{\int_{0}^{\tau_{n}}\left[\Gamma_{t}^{2} \Delta u_{t}+\Gamma_{t}^{3} \Delta v_{t}\right] d t\right\}
$$

Note that $Y_{t}^{2}, \nabla X_{t}, Y_{t}^{1}, \nabla G_{t}$ are continuous, and that

$$
E\left\{\sup _{0 \leq t \leq T}\left[\left|Y_{t}^{2}\right|^{2}+\left|\nabla X_{t}\right|^{2}+\left|Y_{t}^{1}\right|^{2}+\left|\nabla G_{t}\right|^{2}\right]+\int_{0}^{T}\left[\left|\Gamma_{t}^{2}\right|+\left|\Gamma_{t}^{3}\right|\right] d t\right\}<\infty
$$

Let $n \rightarrow \infty$ in (2.44) and apply the dominated convergence theorem to get

$$
E\left\{Y_{T}^{2} \nabla X_{T}-Y_{T}^{1} \nabla G_{T}\right\}=E\left\{\int_{0}^{T}\left[\Gamma_{t}^{2} \Delta u_{t}+\Gamma_{t}^{3} \Delta v_{t}\right] d t\right\},
$$

which, together with (2.39), proves the theorem.

For the future use, note that from the above proof we have the following.

Lemma 2.9. Assume $X_{t}=\int_{0}^{t} \alpha_{s} d W_{s}+A_{t}$ is a continuous semimartingale. Suppose that

(1) $\int_{0}^{T}\left|\alpha_{t}\right|^{2} d t<\infty$, a.s;

(2) both $X_{t}$ and $A_{t}$ are uniformly (in $t$ ) integrable.

Then $E\left[X_{T}\right]=E\left[A_{T}\right]$.

The following theorem is the main result of this section. 
Theorem 2.10 (necessary conditions for optimality). Assume (A1)-(A3). If $\left(\widehat{C}_{T}, \hat{u}, \hat{v}\right) \in$ $A$ is the optimal solution for the problem of maximizing (2.15), then the following maximum conditions hold true, with self-evident notation:

$$
\widehat{\Gamma}_{T}^{1}=0 ; \quad \widehat{\Gamma}_{t}^{2}=0 ; \quad \widehat{\Gamma}_{t}^{3}=0 .
$$

Remark 2.11. (i) If we define the Hamiltonian $H$ as

$$
H\left(t, X_{t}, u_{t}, v_{t}, Y_{t}, Z_{t}\right):=Y_{t}^{2} f\left(t, X_{t}, u_{t}, v_{t}\right)-Y_{t}^{1} g\left(t, X_{t}, u_{t}, v_{t}\right)+Z_{t}^{2} v_{t},
$$

then the last two maximum conditions become

$$
H_{u}\left(t, \hat{X}_{t}, \hat{u}_{t}, \hat{v}_{t}, Y_{t}, Z_{t}\right)=0, \quad H_{v}\left(t, \hat{X}_{t}, \hat{u}_{t}, \hat{v}_{t}, Y_{t}, Z_{t}\right)=0
$$

(ii) The condition $\widehat{\Gamma}_{T}^{1}=0$ is a familiar optimality condition for risk-sharing, that appears in the literature in single-period models.

Proof of Theorem 2.10. Since $\left(\hat{C}_{T}, \hat{u}, \hat{v}\right)$ is optimal, for any bounded $\Delta C_{T}, \Delta u, \Delta v$ and any small $\varepsilon>0$, we have $\hat{J}^{\varepsilon} \leq \hat{J}$ where $\hat{J}^{\varepsilon}$ and $\hat{J}$ are defined in the obvious way. Thus $\nabla \widehat{J}^{\varepsilon} \leq 0$. By Corollary 2.7 we get $\nabla \hat{J} \leq 0$. In particular, for

$$
\Delta C_{T} \triangleq \operatorname{sign}\left(\widehat{\Gamma}_{T}^{1}\right) ; \quad \Delta u_{t} \triangleq \operatorname{sign}\left(\widehat{\Gamma}_{t}^{2}\right) ; \quad \Delta v_{t} \triangleq \operatorname{sign}\left(\nabla \widehat{\Gamma}_{t}^{3}\right),
$$

we have

$$
0 \geq \nabla \hat{J}=E\left\{\left|\hat{\Gamma}_{T}^{1}\right|+\int_{0}^{T}\left[\left|\widehat{\Gamma}_{t}^{2}\right|+\left|\widehat{\Gamma}_{t}^{3}\right|\right] d t\right\}
$$

which obviously proves the theorem.

We next show that the necessary conditions can be written as a coupled forwardbackward SDE. To this end, we note that $\lambda>0$. By (A2) we have

$$
\frac{\partial}{\partial c}\left[\lambda \partial_{x} U_{1}(c, g)-U_{2}^{\prime}(x-c)\right]=\lambda \partial_{x x} U_{1}(c, g)+U_{2}^{\prime \prime}(x-c) \leq 0 .
$$

If we have strict inequality, then there exists a function $F_{1}$ such that for any $x, g$, value $c \triangleq F_{1}(x, g)$ is the solution to the equation

$$
\lambda \partial_{x} U_{1}(c, g)-U_{2}^{\prime}(x-c)=0 .
$$

Similarly, for $y_{1}, y_{2}>0$, and any $(t, x, u, v)$, by (A1) we know that

$$
\left[\begin{array}{cc}
\frac{\partial}{\partial u}\left(y_{2} f_{u}-y_{1} g_{u}\right) & \frac{\partial}{\partial v}\left(y_{2} f_{u}-y_{1} g_{u}\right) \\
\frac{\partial}{\partial u}\left(y_{2} f_{v}-y_{1} g_{v}\right) & \frac{\partial}{\partial v}\left(y_{2} f_{v}-y_{1} g_{v}\right)
\end{array}\right]=y_{2}\left[\begin{array}{ll}
f_{u u} & f_{u v} \\
f_{u v} & f_{v v}
\end{array}\right]-y_{1}\left[\begin{array}{ll}
g_{u u} & g_{u v} \\
g_{u v} & g_{v v}
\end{array}\right]
$$

is negative definite. If it is strictly negative definite, then there exist functions $F_{2}, F_{3}$ such that for any $y_{1}, y_{2}>0$ and any $\left(t, x, z_{2}\right)$, values $u \triangleq F_{2}\left(t, x, y_{1}, y_{2}, z_{2}\right)$ and $v \triangleq F_{3}\left(t, x, y_{1}, y_{2}, z_{2}\right)$ 
are the solution to the system of equations

$$
y_{2} f_{u}(t, x, u, v)-y_{1} g_{u}(t, x, u, v)=0 ; \quad y_{2} f_{v}(t, x, u, v)-y_{1} g_{v}(t, x, u, v)+z_{2}=0 .
$$

Theorem 2.12. Assume (A1)-(A3), that there exist functions $F_{1}, F_{2}, F_{3}$ as above, and that $\left(f_{u} g_{u}\right)(t, x, u, v)>0$ for any $(t, x, u, v)$. If $\left(\hat{C}_{T}, \hat{u}, \hat{v}\right) \in \mathscr{A}$ is the optimal solution for the problem of maximizing (2.15), then $\hat{X}, Y^{1}, Y^{2}, Z^{1}$, and $Z^{2}$ satisfy the following FBSDE:

$$
\begin{gathered}
\hat{G}_{t}=\int_{0}^{t} \hat{g}_{s} d s \\
\hat{X}_{t}=x+\int_{0}^{t} \hat{f}(s) d s+\int_{0}^{t} F_{3}\left(s, \hat{X}_{s}, Y_{s}^{1}, Y_{s}^{2}, Z_{s}^{2}\right) d W_{s} \\
Y_{t}^{1}=-\lambda \partial_{y} U_{1}\left(F_{1}\left(\hat{X}_{T}, \hat{G}_{T}\right), \hat{G}_{T}\right)-\int_{t}^{T} Z_{s}^{1} d W_{s} ; \\
Y_{t}^{2}=U_{2}^{\prime}\left(\hat{X}_{T}-F_{1}\left(\hat{X}_{T}, \hat{G}_{T}\right)\right)-\int_{t}^{T}\left[Y_{s}^{1} \hat{g}_{x}(s)-Y_{s}^{2} \hat{f}_{x}(s)\right] d s-\int_{t}^{T} Z_{s}^{2} d W_{s},
\end{gathered}
$$

where, for $\varphi=f, g, f_{x}, g_{x}$,

$$
\varphi(s) \triangleq \varphi\left(s, X_{s}, F_{2}\left(s, X_{s}, Y_{s}^{1}, Y_{s}^{2}, Z_{s}^{2}\right), F_{3}\left(s, X_{s}, Y_{s}^{1}, Y_{s}^{2}, Z_{s}^{2}\right)\right) .
$$

Moreover, the optimal controls are

$$
\begin{gathered}
\hat{C}_{T}=F_{1}\left(\hat{X}_{T}, \hat{G}_{T}\right) ; \\
\hat{u}_{t}=F_{2}\left(s, \hat{X}_{s}, Y_{s}^{1}, Y_{s}^{2}, Z_{s}^{2}\right) ; \\
\hat{v}_{t}=F_{3}\left(s, \hat{X}_{s}, Y_{s}^{1}, Y_{s}^{2}, Z_{s}^{2}\right) .
\end{gathered}
$$

Proof. By Theorem 2.10 we have (2.47). From $\Gamma_{T}^{1}=0$ we get $\hat{C}_{T}=F_{1}\left(\hat{X}_{T}, \hat{G}_{T}\right)$. Since $\lambda \partial_{y} U_{1}<0$, we have $Y_{t}^{1}>0$. Moreover, by $\Gamma_{t}^{2}=0$ and the assumption that $f_{u} g_{u}>0$ we get $Y_{t}^{2}>0$. Then we have

$$
\begin{aligned}
& \hat{u}_{t}=F_{2}\left(s, \hat{X}_{s}, Y_{s}^{1}, Y_{s}^{2}, Z_{s}^{2}\right) \\
& \hat{v}_{t}=F_{3}\left(s, \hat{X}_{s}, Y_{s}^{1}, Y_{s}^{2}, Z_{s}^{2}\right) .
\end{aligned}
$$

Now by the definition of the processes on the left-hand sides of (2.56), we see that the right-hand sides are true.

Remark 2.13. The existence and uniqueness of FBSDEs is studied, among other papers, in $[6,14]$, and in the monograph by Ma and Yong [9] which contains more references. Since the coefficients of our FBSDE depend on inverse functions $F_{1}, F_{2}, F_{3}$, it does not seem feasible to state a nice set of sufficient conditions under which the existence and uniqueness would be guaranteed. In the examples we solve in Section 4, it turns out that we do not use the FBSDE form (2.56), but we manage to guess the explicit relationship between adjoint processes $Y_{1}$ and $Y_{2}$, express $Z_{2}$ as a function of other variables setting 
$\Gamma^{3} \equiv 0$ in (2.38), then treat equations for $Y_{1}, Y_{2}$ as forward SDEs and the equation for $X$ as the backward SDE. In general, there is no explicit relationship between $Y_{1}$ and $Y_{2}$ and this is not possible.

2.3. Sufficient conditions for optimality. Let us assume that there is a multiple $\left(\widehat{C}_{T}, \hat{X}\right.$, $\left.Y^{1}, Y^{2}, Z^{1}, Z^{2}, \hat{u}, \hat{v}\right)$ that satisfies the necessary conditions of Theorem 2.10. We want to check that those are also sufficient conditions, that is, that $\left(\hat{C}_{T}, \hat{u}, \hat{v}\right)$ is optimal. Here, it is essential to have the concavity of $f$ and $-g$. Let $\left(C_{T}, u, v\right)$ be an arbitrary admissible control triple, with corresponding $X, G$, and we allow now $\varepsilon_{0}=0$ in the assumption (A3). We have

$$
\begin{aligned}
& J\left(\widehat{C_{T}}, \widehat{u}, \widehat{v}\right)-J\left(C_{T}, u, v\right) \\
& \quad=E\left\{\left[U_{2}\left(\widehat{X}_{T}-\hat{C}_{T}\right)-U_{2}\left(X_{T}-C_{T}\right)\right]+\lambda\left[U_{1}\left(\widehat{C}_{T}, \hat{G}_{T}\right)-U_{1}\left(C_{T}, G_{T}\right)\right]\right\} .
\end{aligned}
$$

By concavity of $U_{i}$, the terminal conditions on $Y^{i}$, and from $\widehat{\Gamma}^{1}=0$, or equivalently $\hat{U}_{2}^{\prime}=$ $\lambda \partial_{x} \hat{U}_{1}$, suppressing the arguments of these functions, we get

$$
\begin{aligned}
J\left(\widehat{C_{T}}, \hat{u}, \hat{v}\right)-J\left(C_{T}, u, v\right) & \\
\geq & E\left\{\left[\left(\hat{X}_{T}-X_{T}\right)-\left(\hat{C}_{T}-C_{T}\right)\right] \hat{U}_{2}^{\prime}+\lambda\left[\left(\hat{C}_{T}-C_{T}\right) \partial_{x} \hat{U}_{1}+\left(\hat{G}_{T}-G_{T}\right) \partial_{y} \hat{U}_{1}\right]\right\} \\
= & E\left\{\left(\hat{X}_{T}-X_{T}\right) \hat{U}_{2}^{\prime}+\lambda\left(\hat{G}_{T}-G_{T}\right) \partial_{y} \hat{U}_{1}\right\}=E\left\{Y_{T}^{2}\left(\hat{X}_{T}-X_{T}\right)-Y_{T}^{1}\left(\hat{G}_{T}-G_{T}\right)\right\} \\
= & E\left\{\int _ { 0 } ^ { T } \left[Y_{t}^{2}(\hat{f}(t)-f(t))+Z_{t}^{2}\left(\hat{v}_{t}-v_{t}\right)-Y_{t}^{1}(\hat{g}(t)-g(t))\right.\right. \\
& \left.\left.\quad-\left(\hat{X}_{t}-X_{t}\right)\left(Y_{t}^{2} \hat{f}_{x}(t)-Y_{t}^{1} \hat{g}_{x}(t)\right)\right] d t\right\} \\
=E & \left\{\int _ { 0 } ^ { T } \left[H\left(t, \hat{X}_{t}, \hat{u}_{t}, \hat{v}_{t}, Y_{t}, Z_{t}\right)-H\left(t, X_{t}, u_{t}, v_{t}, Y_{t}, Z_{t}\right)\right.\right. \\
& \left.\left.\quad-\left(\hat{X}_{t}-X_{t}\right) H_{x}\left(t, \hat{X}_{t}, \hat{u}_{t}, \hat{v}_{t}, Y_{t}, Z_{t}\right)\right] d t\right\} .
\end{aligned}
$$

Here, the second to last equality is proved using Itô's rule and the dominated convergence theorem, and in a similar way as in the proof of Theorem 2.8. Note that $Y^{1}$ is positive, as a martingale with positive terminal value. Then, from $Y^{2} \hat{f}_{u}=Y^{1} \hat{g}_{u}$, we know that $Y_{t}^{2}$ is also positive. Since $f$ and $-g$ are concave in $\left(X_{t}, u_{t}, v_{t}\right)$, this implies that $H\left(t, X_{t}, u_{t}, v_{t}, Y_{t}, Z_{t}\right)$ is also concave in $\left(X_{t}, u_{t}, v_{t}\right)$, and we have

$$
\left(\hat{X}_{t}-X_{t}\right) H_{x}\left(t, \hat{X}_{t}, \hat{u}_{t}, \hat{v}_{t}, Y_{t}, Z_{t}\right) \leq H\left(t, \hat{X}_{t}, \hat{u}_{t}, \hat{v}_{t}, Y_{t}, Z_{t}\right)-H\left(t, X_{t}, u_{t}, v_{t}, Y_{t}, Z_{t}\right)
$$

since, by Remark 2.11, $\partial_{u} \hat{H}=\partial_{v} \hat{H}=0$. Thus, $J\left(\hat{C}_{T}, \hat{u}, \hat{v}\right)-J\left(C_{T}, u, v\right) \geq 0$. We have proved the following. 
Theorem 2.14. Under the assumptions of Theorem 2.10, if $\hat{C}_{T}, \hat{u}, \hat{v}, \hat{X}_{t}, Y_{t}^{1}, Y_{t}^{2}, Z_{t}^{1}, Z_{t}^{2}$ satisfy the necessary conditions of Theorem 2.10, and $\left(\hat{C}_{T}, \hat{u}, \hat{v}\right)$ is admissible, possibly with $\varepsilon_{0}=0$, then $\left(\hat{C}_{T}, \hat{u}, \hat{v}\right)$ is an optimal triple for the problem of maximizing $J\left(C_{T}, u, v ; \lambda\right)$.

Remark 2.15. There remains a question of determining the appropriate Lagrange multiplier $\hat{\lambda}$, if it exists. We now describe the usual way for identifying it, without giving assumptions for this method to work. Instead, we refer the reader to Luenberger [8]. First, define

$$
\tilde{V}(\lambda)=\sup _{C, u, v} E\left[U_{2}\left(X_{T}^{u, v}-C_{T}\right)+\lambda U_{1}\left(C_{T}, G_{T}^{u, v}\right)\right]
$$

Then, the appropriate $\hat{\lambda}$ is the one that minimizes $\tilde{V}(\lambda)-\lambda R$, if it exists. If for this $\lambda=\hat{\lambda}$ there exists an optimal control $(\hat{C}, \hat{u}, \hat{v})$ for the problem on the right-hand side of $(2.16)$, and if we have $E\left[U_{1}\left(\hat{C}_{T}, G_{T}^{\hat{u}, \hat{v}}\right)\right]=R$, then $(\hat{C}, \hat{u}, \hat{v})$ is also optimal for the problem on the left-hand side of (2.16).

\section{Implementing the first-best solution}

We now consider the situation in which the agent chooses the controls $(u, v)$. Although the condition $\hat{\Gamma}_{T}^{1}=0$ determines the value of the contract $C_{T}$ at time $T$ as a function of $X_{T}, G_{T}$, typically, it is not true that the contract can be offered as such a function, and still induce the agent to implement optimal $(u, v)$.

We assume that the function $U_{2}^{\prime}$ is a one-to-one function on its domain, with the inverse function denoted

$$
I_{2}(z):=\left(U_{2}^{\prime}\right)^{-1}(z)
$$

Note that the boundary condition $Y_{T}^{2}=U_{2}^{\prime}\left(\hat{X}_{T}-\hat{C}_{T}\right)$ gives another value for $C_{T}, \hat{C}_{T}=$ $\hat{X}_{T}-I_{2}\left(Y_{T}^{2}\right)$. In the problem of executive compensation, this has an interpretation of the executive being payed by the difference between the stock value and a value $I_{2}\left(Y_{T}^{2}\right)$ of a benchmark portfolio. We will see that the contract of this form indeed induces the agent to apply the first-best controls $(\hat{u}, \hat{v})$. We have the following.

Definition 3.1. An admissible triple $\left(\hat{C}_{T}, \hat{u}, \widehat{v}\right)$ is said to be implementable if there exists an implementable contract $F$ such that $u^{F}=\widehat{u}, v^{F}=\hat{v}$ and that $F\left(\omega, X^{\hat{u}, \hat{v}}\right)=\hat{C}$.

Since we assume here that both the agent and the principal have full information, the first-best contract will be implementable since the principal observes $(u, v)$ and can punish the agent if she does not apply the optimal $(u, v)$. However, we now show that the optimal $(u, v)$ can be implemented by a natural contract without direct punishment, which includes the framework studied in Ou-Yang [13].

Proposition 3.2. Assume (A1)-(A3) and suppose that there exists $\hat{\lambda}>0$ so that the necessary conditions are satisfied with $\hat{C}_{T}, \hat{u}, \hat{v}, \hat{X}, \hat{G}, Y^{1}, Y^{2}, Z^{1}, Z^{2}$, that $\left(\hat{C}_{T}, \hat{u}, \hat{v}\right)$ is admissible, and that the IR constraint is satisfied as an equality, that is,

$$
E\left[U_{1}\left(\hat{C}_{T}, \hat{G}_{T}\right)\right]=R
$$


Then, the first-best triple $(\hat{C}, \hat{u}, \hat{v})$ is implementable with the contract

$$
F(\omega, X)=X_{T}-I_{2}\left(Y_{T}^{2}(\omega)\right)
$$

In particular, it is sufficient for the principal to observe $\left\{W_{t}\right\}_{0 \leq t \leq T}$ and $X_{T}$ in order to implement the first-best contract.

Proof. It suffices to show that $(\hat{u}, \hat{v})$ maximizes $E\left[U_{1}\left(X_{T}-I_{2}\left(Y_{T}^{2}\right), G_{T}\right)\right]$. Similarly as above for the principal's problem, denoting by $\tilde{Y}^{i} / \hat{\lambda}, \tilde{Z}^{i} / \hat{\lambda}$ the adjoint processes for the agent's problem, we can verify that the necessary and sufficient conditions for $(\tilde{u}, \tilde{v})$ to be optimal for the agent are given by the system

$$
\begin{gathered}
d \tilde{X}_{t}=f\left(t, \tilde{X}_{t}, \tilde{u}_{t}, \tilde{v}_{t}\right) d t+\tilde{v}_{t} d W_{t}, \quad \tilde{X}_{0}=x, \quad d \tilde{Y}_{t}^{1}=\tilde{Z}_{t}^{1} d W_{t}, \\
d \tilde{Y}_{t}^{2}=\left[\tilde{Y}_{t}^{1} g_{x}\left(t, \tilde{X}_{t}, \tilde{u}_{t}, \tilde{v}_{t}\right)-\tilde{Y}_{t}^{2} f_{x}\left(t, \tilde{X}_{t}, \tilde{u}_{t}, \tilde{v}_{t}\right)\right] d t+\tilde{Z}_{t}^{2} d W_{t}, \\
\tilde{Y}_{T}^{1}=-\hat{\lambda} \partial_{y} U_{1}\left(\tilde{X}_{T}-I_{2}\left(Y_{T}^{2}\right), \tilde{G}_{T}\right), \quad \tilde{Y}_{T}^{2}=\hat{\lambda} \partial_{x} U_{1}\left(\tilde{X}_{T}-I_{2}\left(Y_{T}^{2}\right), \tilde{G}_{T}\right),
\end{gathered}
$$

with the maximum conditions

$$
\begin{gathered}
\tilde{Y}_{t}^{2} f_{u}\left(t, \tilde{X}_{t}, \tilde{u}_{t}, \tilde{v}_{t}\right)=\tilde{Y}_{t}^{1} g_{u}\left(t, \tilde{X}_{t}, \tilde{u}_{t}, \tilde{v}_{t}\right), \\
\tilde{Y}_{t}^{2} f_{v}\left(t, \tilde{X}_{t}, \tilde{u}_{t}, \tilde{v}_{t}\right)+\tilde{Z}_{t}^{2}=\tilde{Y}_{t}^{1} g_{v}\left(t, \tilde{X}_{t}, \tilde{u}_{t}, \tilde{v}_{t}\right) .
\end{gathered}
$$

It is now easy to see that $\hat{C}_{T}, \hat{u}, \hat{v}, \hat{X}, \hat{G}, Y^{i}, Z^{i}$ satisfy this system. By defining $H$ as in Remark 2.11 and noting that $H$ is concave, analogous to the proof of Theorem 2.14 one can show that the pair $(\hat{u}, \hat{v})$ is optimal for the agent. Also, $\hat{C}_{T}=\hat{X}_{T}-I_{2}\left(Y_{T}^{2}\right)=F(\omega, \hat{X})$.

\subsection{Increasing state-contingent compensation and uniqueness of optimal contracts.} We have seen that the contract $F(\omega, X)=X_{T}-I_{2}\left(Y_{T}^{2}\right)$ implements the first-best solution. However, in general, this is not the only contract that does that. In order to get uniqueness, we have to reduce the space of admissible contracts. One natural thing to do is to see whether we can have optimal contracts in the option-like form $F\left(X_{T}\right)$, for some deterministic function $F$, as, for example, in Ross [15]. In Cadenillas et al. [1], sufficient conditions are found under which there is a contract of such a form which attains the maximal possible utility for the principal, although it does not necessarily implement the first-best solution $(\hat{u}, \hat{v}, \widehat{C})$. Moreover, the conditions are quite strong, and in particular, there is no control $u$ of the drift. We generalize their result in Example 4.7. In general, it is not possible to implement the first-best solution with the contracts of the form $F\left(X_{T}\right)$. Instead, we consider the following contracts.

Definition 3.3. A contract is said to be of the increasing state-contingent compensation (ISCC) type if it is of the form $F\left(X_{T}\right)-D_{T}$, where $F(x)$ is a deterministic function, such that $F^{\prime}(x)>0$, and $D_{T}$ is a $\mathscr{F}_{T}$ measurable random variable.

The contract $F(\omega, X)=X_{T}-I_{2}\left(Y_{T}^{2}\right)$ is of the ISCC type, and we will show that it the only contract which implements the first-best solution in that family. As mentioned 
above, the first-best contract can be implemented by a "dictatorial" contract, where the agent gets penalized by negative compensation if he does not use the first-best control. In practice, it is more natural, and consistent with real applications, to consider contracts of the ISCC type, where the payoff depends on the performance of the underlying process $X$ compared to a benchmark. We have the following uniqueness result.

Proposition 3.4. Under the assumptions of Proposition 3.2 and if $\operatorname{range}\left(\hat{X}_{T}\right)=\mathbb{R}$ and $f_{u} g_{u}>0$, the contract $F(\omega, X)=X_{T}-I_{2}\left(Y_{T}^{2}\right)$ is the only ISCC type contract that implements the first-best solution.

Proof. Introduce the agent's objective function, given a contract $F\left(X_{T}\right)-D_{T}$ :

$$
\begin{gathered}
A(u, v) \triangleq E U_{1}\left(F\left(X_{T}\right)-D_{T}, G_{T}\right) ; \\
A^{\varepsilon} \triangleq A\left(u^{\varepsilon}, v^{\varepsilon}\right) ; \quad A \triangleq A(u, v) ; \quad \nabla A^{\varepsilon} \triangleq \frac{A^{\varepsilon}-A}{\varepsilon},
\end{gathered}
$$

where $u^{\varepsilon}, v^{\varepsilon}$ are defined as earlier. Similarly as before, we can check that

$$
\nabla A \triangleq \lim _{\varepsilon \rightarrow 0} \nabla A^{\varepsilon}=E\left\{\partial_{x} U_{1}\left(F\left(X_{T}\right)-D_{T}, G_{T}\right) F^{\prime}\left(X_{T}\right) \nabla X_{T}+\partial_{y} U_{1}\left(F\left(X_{T}\right)-D_{T}, G_{T}\right) \nabla G_{T}\right\}
$$

We define the following two adjoint processes via BSDEs:

$$
\begin{aligned}
K_{t}^{1}= & -\partial_{y} U_{1}\left(F\left(X_{T}\right)-D_{T}, G_{T}\right)-\int_{t}^{T} L_{s}^{1} d W_{s} ; \\
K_{t}^{2}= & \partial_{x} U_{1}\left(F\left(X_{T}\right)-D_{T}, G_{T}\right) F^{\prime}\left(X_{T}\right) \\
& -\int_{t}^{T}\left[K_{s}^{1} g_{x}\left(s, X_{s}, u_{s}, v_{s}\right)-K_{s}^{2} f_{x}\left(s, X_{s}, u_{s}, v_{s}\right)\right] d s-\int_{t}^{T} L_{s}^{2} d W_{s} .
\end{aligned}
$$

Then, we can also check similarly as before that

$$
\nabla A=E\left\{\int_{0}^{T}\left(K_{s}^{2} f_{u}(s)-K_{s}^{1} g_{u}(s)\right) \Delta u_{s} d s+\int_{0}^{T}\left(K_{s}^{2} f_{v}(s)-K_{s}^{1} g_{v}(s)+L_{s}^{2}\right) \Delta v_{s} d s\right\}
$$

At the optimal $(u, v)$, we have $\nabla A \leq 0$. Assume now that the contract $F\left(X_{T}\right)-D_{T}$ implements the first-best solution, so that $\left(\hat{u}, \hat{v}, \hat{X}_{T}\right)$ is the optimal solution to the agent's problem, and $F\left(\hat{X}_{T}\right)-D_{T}=\hat{C}_{T}$. From $\nabla A \leq 0$, we get

$$
\frac{K_{t}^{2}}{K_{t}^{1}}=\frac{g_{u}\left(t, \hat{X}_{t}, \hat{u}, \hat{v}\right)}{f_{u}\left(t, \hat{X}_{t}, \hat{u}, \hat{v}\right)}
$$

for almost all $(t, \omega)$, then by continuity also for $t=T$. Comparing with the necessary condition $\Gamma_{t}^{2}=0$ (see (2.38)), we have

$$
\frac{K_{T}^{2}}{K_{T}^{1}}=\frac{Y_{T}^{2}}{Y_{T}^{1}}
$$


Then, from (2.35) and (3.8), we obtain

$$
F^{\prime}\left(\hat{X}_{T}\right)=\frac{-K_{T}^{2} \partial_{y} U_{1}\left(\hat{C}_{T}, \hat{G}_{T}\right)}{K_{T}^{1} \partial_{x} U_{1}\left(\hat{C}_{T}, \hat{G}_{T}\right)}=\frac{-Y_{T}^{2} \partial_{y} U_{1}\left(\hat{C}_{T}, \hat{G}_{T}\right)}{Y_{T}^{1} \partial_{x} U_{1}\left(\hat{C}_{T}, \hat{G}_{T}\right)}=1
$$

Thus, we can write

$$
F(x)=x+b
$$

for some constant $b$. Since we assume $F\left(\hat{X}_{T}\right)-D_{T}=\hat{C}_{T}$, we get

$$
D_{T}-b=I_{2}\left(Y_{T}^{2}\right)
$$

\section{Examples}

Example 4.1 (one-dimensional, linear dynamics). We solve here one of the problems considered in Cadenillas et al. [1], and solved therein using a different approach. All other examples solved in that paper can also be solved using the approach of this paper, and in a more straightforward way, if we account for the modification of Remark 2.3. We are given

$$
d X_{t}=f\left(u_{t}\right) d t+\alpha v_{t} d t+v_{t} d W_{t}
$$

and $G_{T}=\int_{0}^{T} g\left(u_{t}\right) d t$, where $W$ is one-dimensional. If $f=g=0$, this is the case of portfolio management. We further discuss this case (also with a nonzero cost function $g(t, X, u$, $v)$ ), in more detail in examples below. The agent's utility is nonseparable, $U_{1}(x, y)=$ $U_{1}(x-y)$. We denote $I_{1}(z):=\left(U_{1}^{\prime}\right)^{-1}(z)$. If there is a control $u$ and penalty $G_{T}$ on it, the corresponding necessary and sufficient conditions are

$$
\begin{gathered}
d \hat{X}_{t}=f\left(\hat{u}_{t}\right) d t+\alpha \hat{v}_{t} d t+\hat{v}_{t} d W_{t}, \quad \hat{X}_{0}=x, \\
d Y_{t}^{1}=Z_{t}^{1} d W_{t}, \quad d Y_{t}^{2}=Z_{t}^{2} d W_{t}, \\
Y_{T}^{1}=Y_{T}^{2}=U_{2}^{\prime}\left(\hat{X}_{T}-\hat{C}_{T}\right)=\hat{\lambda} U_{1}^{\prime}\left(\hat{C}_{T}-\hat{G}_{T}\right) .
\end{gathered}
$$

We see that $Y^{1}=Y^{2}, Z^{1}=Z^{2}$, so that the maximum conditions become

$$
f^{\prime}(\widehat{u})=g^{\prime}(\widehat{u}), \quad \alpha Y_{t}^{2}=-Z_{t}^{2} .
$$

(If there is no control $u$, then $Y^{1}=0$.) We assume that the first equality above has a unique solution $\hat{u}$, which is then constant. The second equality gives $Y_{t}^{2}=z \hat{\lambda} \exp \left\{-(1 / 2) \alpha^{2} t-\right.$ $\left.\alpha W_{t}\right\}$ for some $z>0$, to be determined below. The optimal contract should be of the form

$$
\hat{C}_{T}=\hat{X}_{T}-I_{2}\left(Y_{T}^{2}\right)=I_{1}\left(\frac{Y_{T}^{2}}{\hat{\lambda}}\right)+\hat{G}_{T} .
$$


The value of $z$ has to be chosen so that $E\left[U_{1}\left(I_{1}\left(Y_{T}^{2} / \hat{\lambda}\right)\right)\right]=R$, assuming such a value $z$ exists. Denote $Z_{t}:=Y_{t}^{2} / z \hat{\lambda}$. Then $Z$ is a martingale with $Z_{0}=1$. We have

$$
d\left(Z_{t} \hat{X}_{t}\right)=Z_{t}\left[f(\widehat{u}) d t+\hat{v}_{t} d W_{t}\right]
$$

so that $Z_{t} \hat{X}_{t}-\int_{0}^{t} Z_{s} f(\hat{u}) d s$ has to be a martingale. The above system of necessary and sufficient conditions will have a solution if we can find $\hat{v}$ using the martingale representation theorem, where we now consider the equation for $Z \hat{X}$ as a Backward SDE having a terminal condition

$$
Z_{T} \hat{X}_{T}=Z_{T}\left\{I_{2}\left(z \hat{\lambda} Z_{T}\right)+I_{1}\left(z Z_{T}\right)+\hat{G}_{T}\right\}
$$

This BSDE has a solution which satisfies $\hat{X}_{0}=x$ if and only if there is a solution $\hat{\lambda}>0$ of the equation

$$
x=E\left[Z_{T} \hat{X}_{T}\right]-f(\hat{u}) T=E\left[Z_{T}\left\{I_{2}\left(z \hat{\lambda} Z_{T}\right)+I_{1}\left(z Z_{T}\right)+\hat{G}_{T}\right\}\right]-f(\hat{u}) T .
$$

This is indeed the case in examples with exponential utilities $U_{i}(x)=-\left(1 / \gamma_{i}\right) e^{-\gamma_{i} x}, \gamma_{i}>0$, solved in Cadenillas et al. [1]. It is straightforward in this case to check that the solution obtained by the method of this example is indeed admissible. The same is true for power utilities, $U_{i}(x)=\left(1 / \gamma_{i}\right) x^{\gamma_{i}}, \gamma_{i}<1$, if we account for Remark 2.3.

Example 4.2 (no drift control). We consider now an example with no control $u$ of the drift, thus $f_{u}=g=0$. We call this case the risk-sharing model. More precisely,

$$
d X_{t}=f\left(t, X_{t}, v_{t}\right) d t+v_{t} d W_{t}
$$

where $W$ is one-dimensional. Assume that the contract $F\left(X_{T}\right)-D_{T}$ implements the firstbest solution. In (3.8) we have $K_{t}^{1}=L_{t}^{1}=0$, and

$$
K_{t}^{2}=U_{1}^{\prime}\left(F\left(X_{T}\right)-D_{T}\right) F^{\prime}\left(X_{T}\right)+\int_{t}^{T}\left[K_{s}^{2} f_{x}\left(s, X_{s}, v_{s}\right)\right] d s-\int_{t}^{T} L_{s}^{2} d W_{s}
$$

The necessary condition for the agent is

$$
\frac{L_{t}^{2}}{K_{t}^{2}}=-f_{v}\left(t, \hat{X}_{t}, \hat{v}\right)
$$

and, from $\Gamma^{3}=0$ (see (2.38)), we also get

$$
\frac{Z_{t}^{2}}{Y_{t}^{2}}=-f_{v}\left(t, \hat{X}_{t}, \hat{v}\right)
$$


Solving the equations of $K^{2}$ and $Y^{2}$ we see that

$$
\begin{aligned}
K_{T}^{2} & =U_{1}^{\prime}\left(F\left(X_{T}\right)-D_{T}\right) F^{\prime}\left(X_{T}\right) \\
& =K_{0} \exp \left\{\int_{0}^{T}\left[f_{x}\left(t, \hat{X}_{t}, \hat{v}_{t}\right)-\frac{1}{2}\left\|f_{v}\left(t, \hat{X}_{t}, \hat{v}\right)\right\|^{2}\right] d t+\int_{0}^{T} f_{v}\left(t, \hat{X}_{t}, \hat{v}\right) d W_{t}\right\}, \\
Y_{T}^{2} & =\hat{\lambda} U_{1}^{\prime}\left(\hat{C}_{T}\right) \\
& =\hat{\lambda} y_{\hat{\lambda}} \exp \left\{\int_{0}^{T}\left[f_{x}\left(t, \hat{X}_{t}, \hat{v}_{t}\right)-\frac{1}{2} \int_{0}^{T}\left\|f_{v}\left(t, \hat{X}_{t}, \hat{v}\right)\right\|^{2}\right] d t+\int_{0}^{T} f_{v}\left(t, \hat{X}_{t}, \hat{v}\right) d W_{t}\right\}
\end{aligned}
$$

for some constants $K_{0}, y_{\hat{\lambda}}$. From $\hat{C}_{T}=F\left(\hat{X}_{T}\right)-D_{T}$, we have

$$
F^{\prime}\left(\hat{X}_{T}\right)=\frac{K_{T}^{2}}{U_{1}^{\prime}\left(F\left(\hat{X}_{T}\right)-D_{T}\right)}=\frac{K_{T}^{2}}{Y_{T}^{2} / \hat{\lambda}}=\frac{K_{0}}{y_{\hat{\lambda}}} .
$$

This means, if range $\left(\hat{X}_{T}\right)=\mathbb{R}$, we can write

$$
F(x)=k x+b
$$

for some positive constant $k$, which is arbitrary. From

$$
I_{1}\left(\frac{1}{\hat{\lambda}} Y_{T}^{2}\right)=\hat{C}_{T}=k \hat{X}_{T}+b-D_{T}=k\left(\left[I_{1}\left(\frac{1}{\hat{\lambda}} Y_{T}^{2}\right)+I_{2}\left(Y_{T}^{2}\right)\right]\right)+b-D_{T},
$$

we have

$$
b-D_{T}=I_{1}\left(\frac{1}{\hat{\lambda}} Y_{T}^{2}\right)-k\left[I_{1}\left(\frac{1}{\hat{\lambda}} Y_{T}^{2}\right)+I_{2}\left(Y_{T}^{2}\right)\right] .
$$

This gives us the necessity part of the following proposition.

PROPOSITION 4.3. In the risk-sharing model described above, if range $\left(\hat{X}_{T}\right)=\mathbb{R}$, if an ISCCtype contract can implement the first-best solution, then it takes the form

$$
F\left(X_{T}\right)-D_{T}=k X_{T}+I_{1}\left(\frac{1}{\hat{\lambda}} Y_{T}^{2}\right)-k\left[I_{1}\left(\frac{1}{\hat{\lambda}} Y_{T}^{2}\right)+I_{2}\left(Y_{T}^{2}\right)\right]
$$

with any positive number $k$. If in addition $f(t, x, v)=\alpha v$, then any such contract, that also satisfies the IR constraint, implements the first-best solution.

Proof. It remains to show sufficiency, that is, the last statement. In this linear model we have $f_{x}=0, f_{v}=\alpha$, so that, from (4.12), the ratio $K_{T}^{2} / Y_{T}^{2}$ is a constant. We then get, from (4.9), that

$$
F\left(X_{T}\right)-D_{T}=I_{1}\left(\frac{1}{k} K_{T}^{2}\right)=I_{1}\left(c Y_{T}^{2}\right)
$$


for some constants $c_{1}, c$. However, this constant is determined uniquely from the IR constraint, that is, $c=1 / \hat{\lambda}$. Thus, substituting $F\left(X_{T}\right)-D_{T}$ from (4.17) in (4.18), we get

$$
X_{T}=I_{1}\left(\frac{1}{\hat{\lambda}} Y_{T}^{2}\right)+I_{2}\left(Y_{T}^{2}\right)
$$

which is the same as the first-best.

Notice that with $f_{u}=g=0$, the increasing state-contingent compensation is not quite unique, unlike the case of Proposition 4.3.

We now have the following result.

COROLlary 4.4. If there exists a positive number $\hat{k}$, such that

$$
I_{1}\left(\frac{1}{\hat{\lambda}} Y_{T}^{2}\right)-\hat{k}\left[I_{1}\left(\frac{1}{\hat{\lambda}} Y_{T}^{2}\right)+I_{2}\left(Y_{T}^{2}\right)\right]=m
$$

for some constant $m$, then the first-best solution can be implemented by the linear contract

$$
H\left(X_{T}\right)=\hat{k} X_{T}+m
$$

if the IR constraint is satisfied under this contract.

Example 4.5 (semilinear drift and cost, exponential utilities and linear contracts). Consider now this generalization of the previous example:

$$
d X_{t}=\left[r_{t} X_{t}+f\left(t, u_{t}, v_{t}\right)\right] d t+v_{t} d W_{t}
$$

where $f$ is a deterministic function of $(t, u, v)$ and $r_{t}$ is a given deterministic process. Assume exponential utility functions, $U_{i}(x)=-\left(1 / R_{i}\right) \exp \left\{-R_{i} x\right\}$. Also assume, with a slight abuse of notation $g$, that

$$
g\left(t, X_{t}, u_{t}, v_{t}\right)=\mu_{t} X_{t}+g\left(t, u_{t}, v_{t}\right)
$$

where $g$ is a deterministic function of $(t, u, v)$, and $\mu_{t}$ is a given deterministic process. Denote

$$
\gamma_{t, s}:=e^{\int_{s}^{t} r_{u} d u}, \quad \gamma_{t}:=e^{\int_{0}^{t} r_{u} d u}
$$

Then, it is seen that

$$
X_{t}=X_{0}+\int_{0}^{t} \gamma_{t, s} f\left(s, u_{s}, v_{s}\right) d s+\int_{0}^{t} \gamma_{t, s} v_{s} d W_{s}
$$

We want to show that the optimal $\hat{u}, \hat{v}$ are deterministic, and we want to see under what conditions we can offer a linear contract. 
The adjoint processes $Y^{1}, Y^{2}$ satisfy

$$
\begin{gathered}
Y_{t}^{1}=\lambda U_{1}^{\prime}\left(C_{T}-G_{T}\right)-\int_{t}^{T} Z_{s}^{1} d W_{s}, \\
Y_{t}^{2}=U_{2}^{\prime}\left(X_{T}-C_{T}\right)-\int_{t}^{T}\left(Y_{s}^{1} \mu_{s}-Y_{s}^{2} r_{s}\right) d s-\int_{t}^{T}\left[Y_{s}^{1} \tilde{g}_{v}\left(s, \hat{u}_{s}, \hat{v}_{s}\right)-Y_{s}^{2} f_{v}\left(s, \hat{u}_{s}, \hat{v}_{s}\right)\right] d W_{s} .
\end{gathered}
$$

The maximum conditions give $Y_{T}^{1}=Y_{T}^{2}$.

We conjecture that optimal $\hat{u}, \hat{v}$ are deterministic processes, and that

$$
Y_{t}^{1}=h_{t} Y_{t}^{2}, \quad h_{T}=1,
$$

where $h$ is a deterministic function of time. Using Itô's rule, it is easily verified that the above equality is satisfied if $h$ is a solution to the ODE:

$$
h_{t}\left(h_{t} \mu_{t}-r_{t}\right)=-h_{t}^{\prime}, \quad h_{T}=1
$$

We assume that $\mu$ and $r$ are such that a unique solution exists. We get one equation for $\hat{u}$, $\hat{v}$ by setting $\Gamma^{2}=0($ see $(2.38))$ :

$$
f_{u}\left(t, \hat{u}_{t}, \hat{v}_{t}\right)=h g_{u}\left(t, \hat{u}_{t}, \hat{v}_{t}\right) .
$$

Note that we can solve for $Y^{2}$ as

$$
\begin{aligned}
Y_{t}^{2}=Y_{0}^{2} \exp \left\{\int_{0}^{t}\left[h_{s} \mu_{s}-r_{s}-\frac{1}{2}\left\|h_{s} g_{v}\left(s, \hat{u}_{s}, \hat{v}_{s}\right)-f_{v}\left(s, \hat{u}_{s} \hat{v}_{s}\right)\right\|^{2}\right] d t\right. \\
\left.+\int_{0}^{t}\left[h_{s} g_{v}\left(s, \hat{u}_{s}, \hat{v}_{s}\right)-f_{v}\left(s, \hat{u}_{s}, \hat{v}_{s}\right)\right] d W_{s}\right\} .
\end{aligned}
$$

Thus, the process

$$
\begin{aligned}
A_{t} & =\log \left(\frac{Y_{t}^{2}}{Y_{0}^{2}}\right)-\int_{0}^{t}\left[h_{s} \mu_{s}-r_{s}-\frac{1}{2}\left\|h_{s} g_{v}\left(s, \hat{u}_{s}, \hat{v}_{s}\right)-f_{v}\left(s, \hat{u}_{s}, \hat{v}_{s}\right)\right\|^{2}\right] d t \\
& =\int_{0}^{t}\left[h_{s} g_{v}\left(s, \hat{u}_{s}, \hat{v}_{s}\right)-f_{v}\left(s, \hat{u}_{s}, \hat{v}_{s}\right)\right] d W_{s}
\end{aligned}
$$

is a local martingale. We conjecture that it is a martingale for the optimal $\hat{u}, \hat{v}$.

We will need below this representation for the first term of $G_{T}$, obtained by integration by parts:

$$
\int_{0}^{T} \mu_{s} X_{s} d s=\frac{X_{T}}{\gamma_{T}} \int_{0}^{T} \mu_{s} \gamma_{s} d s-\int_{0}^{T} \int_{0}^{s} \mu_{u} \gamma_{u} d u\left[f\left(s, \hat{u}_{s}, \hat{v}_{s}\right) \gamma_{s}^{-1} d s+\gamma_{s}^{-1} \hat{v}_{s} d W_{s}\right]
$$

From the maximum condition $\lambda U_{1}^{\prime}\left(C_{T}-G_{T}\right)=U_{2}^{\prime}\left(X_{T}-C_{T}\right)$, we can verify that

$$
X_{T}-C_{T}=a+b X_{T}-b G_{T}, \quad a=\frac{\log (\lambda)}{R_{1}+R_{2}}, b=\frac{R_{1}}{R_{1}+R_{2}} .
$$


From this and $Y_{T}^{2}=U_{2}^{\prime}\left(X_{T}-C_{T}\right)$ we get

$$
\log Y_{T}^{2}=-a R_{2}+b R_{2}\left(G_{T}-X_{T}\right)
$$

From this, using the fact that $\hat{v}$ is assumed deterministic, denoting by $d$ a generic constant, we can get an alternative representation of $A_{t}$ as

$$
A_{t}=E_{t}\left[A_{T}\right]=d+b R_{2} E_{t}\left[G_{T}-X_{T}\right]
$$

Using (4.32) and that the nondeterministic part of $G_{T}$ is $\int_{0}^{T} \mu_{u} X_{u} d u$, we get

$$
A_{t}=d+b R_{2}\left(\frac{\int_{0}^{T} \mu_{s} \gamma_{s} d s}{\gamma_{T}}-1\right) E_{t}\left[X_{T}\right]-b R_{2} \int_{0}^{t}\left(\int_{0}^{s} \mu_{u} \gamma_{u} d u\right) \gamma_{s} \hat{v}_{s} d W_{s}
$$

Since $E_{t}\left[X_{T}\right]=X_{0}+\int_{0}^{t} \gamma_{T, s} \hat{v}_{s} d W_{s}$, comparing the $d W$ integrand in this last expression to the $d W$ integrand in $(4.31)$, we see that we need to have

$$
\begin{aligned}
h_{s} g_{v}\left(s, \hat{u}_{s}, \hat{v}_{s}\right)-f_{v}\left(s, \hat{u}_{s}, \hat{v}_{s}\right) & =b R_{2}\left(\frac{\int_{0}^{T} \mu_{s} \gamma_{s} d s}{\gamma_{T}}-1\right) \gamma_{T, s} \hat{v}_{s}-b R_{2}\left(\int_{0}^{s} \mu_{u} \gamma_{u} d u\right) \gamma_{s}^{-1} \hat{v}_{s} \\
& =\gamma_{s}^{-1} \hat{v}_{s} b R_{2}\left(\int_{s}^{T} \mu_{u} \gamma_{u} d u-\gamma_{T}\right) .
\end{aligned}
$$

We assume that $g$ and $f$ are such that this equation together with (4.29) has a unique solution $\left(\hat{u}_{s}, \hat{v}_{s}\right)$ for all $s$, and such that in the above calculations the local martingales are, indeed, martingales. Then, we have shown that our conjecture of deterministic optimal controls was correct, and $(\hat{u}, \hat{v})$ is the first-best solution.

Remark 4.6. We can get the problem solved in Ou-Yang [13] as a special case of this example. (That paper considers a model with a multidimensional Brownian motion, and we could modify our example to deal with that case.) In that problem,

$$
f(t, u, v)=\alpha v, \quad g(t, u, v)=\frac{k_{t} v^{2}}{2}, \quad r_{t} \equiv r, \quad \mu_{t} \equiv \mu,
$$

where $\mu, r$ are constant, and $k_{t}$ is a deterministic function of time. Then, we can compute from (4.28) and (4.37)

$$
h_{t}=\left[\frac{\mu}{r}+\left(\frac{1-\mu}{r}\right) e^{r(T-t)}\right]^{-1}, \quad \hat{v}_{t}=\frac{\alpha h_{t}}{b R_{2}+k_{t} h_{t}^{2}} .
$$

Knowing this we also know $Y_{t}^{2}$, and the optimal contract $\hat{C}_{T}=X_{T}-I_{2}\left(Y_{T}^{2}\right)$.

We still want to see under what conditions we can offer a linear contract, using similar arguments as in Proposition 4.3 and Corollary 4.4. Let us first see under what conditions the contract

$$
C_{k}:=k X_{T}+(1-k) I_{1}\left(\frac{Y_{T}^{2}}{\lambda}\right)-k I_{2}\left(Y_{T}^{2}\right)
$$


implements the first-best solution $\hat{v}$ for any positive $k$. If the agent is given the contract $C_{k}$, the corresponding adjoint equations are

$$
\begin{gathered}
K_{t}^{1}=U_{1}^{\prime}\left(C_{k}-G_{T}\right)-\int_{t}^{T} L_{s}^{1} d W_{s}, \\
K_{t}^{2}=k U_{1}^{\prime}\left(C_{k}-G_{T}\right)-\int_{t}^{T}\left(K_{s}^{1} \mu_{s}-K_{s}^{2} r_{s}\right) d s-\int_{t}^{T}\left[K_{s}^{1} g_{v}\left(s, u_{s}, v_{s}\right)-K_{s}^{2} f_{v}\left(s, u_{s}, v_{s}\right)\right] d W_{s} .
\end{gathered}
$$

We see that $K_{T}^{2}=k K_{T}^{1}$.

Similarly as before, we conjecture that the agent's optimal $v$ is a deterministic process, and we can see that

$$
K_{t}^{1}=\beta_{t} K_{t}^{2}, \quad \beta_{T}=\frac{1}{k}
$$

where $\beta$ is a deterministic function of time, with $\beta$ being a solution to the ODE (4.28), but with $\beta_{T}=1 / k$. We can solve for $K^{2}$ as

$$
\begin{gathered}
K_{t}^{2}=K_{0}^{2} \exp \left\{\int_{0}^{t}\left[\beta_{s} \mu_{s}-r_{s}-\frac{1}{2}\left\|\beta_{s} g_{v}\left(s, u_{s}, v_{s}\right)-f_{v}\left(s, u_{s}, v_{s}\right)\right\|^{2}\right] d t\right. \\
\left.+\int_{0}^{t}\left[\beta_{s} g_{v}\left(s, u_{s}, v_{s}\right)-f_{v}\left(s, u_{s}, v_{s}\right)\right] d W_{s}\right\} .
\end{gathered}
$$

On the other hand, from $K_{T}^{2}=k U_{1}^{\prime}\left(C_{k}-G_{T}\right)$ we get

$$
K_{T}^{2}=k \exp \left\{-R_{1}\left[k X_{T}+(1-k) I_{1}\left(\frac{Y_{T}^{2}}{\lambda}\right)-k I_{2}\left(Y_{T}^{2}\right)-G_{T}\right]\right\} .
$$

Substituting here $I_{i}(y)=-\log (y) / R_{i}$, the expression (4.25) for $X_{T}$, the expression (4.32) for the random part of $G_{T}$, and the expression (4.30) for $Y_{T}^{2}$, and comparing the random terms with the one in (4.43), we see that we need to have

$$
\begin{aligned}
\beta_{s} g_{v}\left(s, u_{s}, v_{s}\right)-f_{v}\left(s, u_{s}, v_{s}\right) \\
=\left(1-k-k \frac{R_{1}}{R_{2}}\right)\left[h_{s} g_{v}\left(s, \hat{u}_{s}, \hat{v}_{s}\right)-f_{v}\left(s, \hat{u}_{s}, \hat{v}_{s}\right)\right] \\
\quad+R_{1}\left(\frac{\int_{0}^{T} \mu_{s} \gamma_{s} d s}{\gamma_{T}}-k\right) \gamma_{T, s} v_{s}-R_{1}\left(\int_{0}^{s} \mu_{u} \gamma_{u} d u\right) \gamma_{s} \hat{v}_{s} \\
=\left(1-k-k \frac{R_{1}}{R_{2}}\right)\left[h_{s} g_{v}\left(s, \hat{u}_{s}, \hat{v}_{s}\right)-f_{v}\left(s, \hat{u}_{s}, \hat{v}_{s}\right)\right]+R_{1} \frac{v_{s}}{\gamma_{s}}\left(\int_{s}^{T} \mu_{u} \gamma_{u} d u-k \gamma_{T}\right) .
\end{aligned}
$$

We want to see under what conditions $u=\hat{u}, v=\hat{v}$, where $(\hat{u}, \hat{v})$ is the first-best solution determined by (4.29) and (4.37). We can easily verify that this happens if there is no control $u, g=0$, and $\mu=0$. In other words, if the cost function is zero and there is no drift control, then the contract $C_{k}$ with any $k>0$ implements the first-best solution. 
Let us now show that, if $g=0, \mu=0$, we can use Corollary 4.4 to make $C_{k}$ linear for some $k=\hat{k}$. We have $I_{i}(x)=-\left(1 / R_{i}\right) \log (x)$. Introduce a deterministic process

$$
\delta_{s}:=h_{s} g_{v}\left(s, \hat{v}_{s}\right)-f_{v}\left(s, \hat{v}_{s}\right)
$$

We want to have

$$
m=-\frac{1}{R_{1}} \log \left(\frac{1}{\hat{\lambda}} Y_{T}^{2}\right)-\hat{k}\left[-\frac{1}{R_{1}} \log \left(\frac{1}{\hat{\lambda}} Y_{T}^{2}\right)-\frac{1}{R_{2}} \log \left(Y_{T}^{2}\right)\right],
$$

or

$$
\begin{aligned}
m= & \frac{1-k}{R_{1}}\left[\log \left(\frac{\hat{\lambda}}{Y_{0}^{2}}\right)+\int_{0}^{T}\left\{h_{s} \mu_{s}-r_{s}+\frac{1}{2} \delta_{s}^{2}\right\} d s\right] \\
& -\frac{k}{R_{2}}\left[\int_{0}^{T}\left\{h_{s} \mu_{s}-r_{s}+\frac{1}{2} \delta_{s}^{2}\right\} d s\right]+\left[\frac{1-k}{R_{1}}-\frac{k}{R_{2}}\right] \int_{0}^{T} \delta_{s} d W_{s},
\end{aligned}
$$

which is satisfied if

$$
\frac{(1-\hat{k})}{R_{1}}-\frac{\hat{k}}{R_{2}}=0,
$$

that is,

$$
\hat{k}=\frac{R_{2}}{R_{1}+R_{2}} .
$$

By Proposition 4.3 and Corollary 4.4, in this model with $g=0, \mu=0$, contract $C_{\hat{k}}$ is linear and it implements the first-best solution.

Example 4.7 (deterministic, option-like contracts). Consider again the model with $f=$ $\alpha v$, where $v$ may be $d$-dimensional. Let us write

$$
Y_{t}:=Y_{t}^{2}=\hat{y} \exp \left\{-\alpha W_{t}-\frac{t\|\alpha\|^{2}}{2}\right\}=: \hat{y} Z_{t}
$$

for some $\hat{y}>0$, where $Z$ is a notation for the exponential martingale process. From (3.8) we see that the process $K^{2}$ is of the form

$$
K_{t}:=K_{t}^{2}=\hat{k} \exp \left\{-\alpha W_{t}-\frac{t\|\alpha\|^{2}}{2}\right\}=\hat{k} Z_{t}
$$

for some number $\hat{k}$. We can show then a multidimensional generalization of this result from Cadenillas et al. [1] about the optimal contract being solely a function $F\left(X_{T}\right)$ of the terminal value $X_{T}$.

Proposition 4.8. Consider the model with $f(t, x, u, v)=\alpha v$. Assume that there exists a function $F(x)=F(x ; \hat{z})$ which satisfies the ordinary differential equation (ODE)

$$
U_{1}^{\prime}(F(x)) F^{\prime}(x)=\frac{\hat{z}}{\hat{y}} U_{2}^{\prime}(x-F(x)),
$$


where $\hat{y}=Y_{0}^{2}$, and $\hat{z}$ is a given constant. Assume that there is a unique function $x(y)=$ $x(y ; \hat{z})$ such that

$$
U_{1}^{\prime}(F(x(y))) F^{\prime}(x(y))=y .
$$

Also assume that $\hat{z}$ and the boundary condition for the solution $F(x)$ of the above ODE can be chosen so that $\hat{z}$ is a unique solution of

$$
E\left[Z_{T} x\left(\widehat{z} Z_{T}\right)\right]=X_{0}
$$

and so that

$$
E\left[U_{1}\left(F\left(x\left(\hat{z} Z_{T}\right)\right)\right)\right]=R .
$$

Then, the principal will attain the first-best utility with the contract $F\left(X_{T}\right)$.

Proof. Given such a contract, it can be shown as before that the agent's optimal process $\hat{X}$ is related to the adjoint process $K^{2}$ of (3.8), from which we get

$$
X_{T}=x\left(\hat{k} Z_{T}\right) .
$$

It is easy to check that the product process $Z X$ is a martingale, from which it follows $\hat{k}=\hat{z}$, comparing to (4.55). From ODE (4.53) and (4.54), we then get

$$
U_{2}^{\prime}\left(X_{T}-F\left(X_{T}\right)\right)=\hat{y} Z_{T}
$$

or

$$
X_{T}-F\left(X_{T}\right)=I_{2}\left(\hat{y} Z_{T}\right) .
$$

But then the principal's utility is equal to

$$
E\left[U_{2}\left(X_{T}-F\left(X_{T}\right)\right)\right]=E\left[U_{2}\left(I_{2}\left(Y_{T}\right)\right)\right],
$$

which is the first-best.

Remark 4.9. Larsen [7] computes numerically an optimal contract in this context, with power utilities, using a different approach. We provide here an alternative way to find an optimal contract, namely solving the above ODE. This would again have to be done numerically.

\section{Conclusions}

We have built a fairly general theory for contracting problems in models driven by Brownian motion, and with full information. A question still remains under which general conditions the optimal contract exists. The recent popular application is the optimal compensation of executives. In order to have more realistic framework for that application, other forms of compensation should be considered, such as a possibility for the agent to cash in the contract at a random time (compensation of American options type). We leave these problems for future research. 


\section{Acknowledgment}

The research is supported in part by NSF Grants DMS 00-99549 and DMS 04-03575.

\section{References}

[1] A. Cadenillas, J. Cvitanić, and F. Zapatero, Optimal risk-sharing with effort and project choice, to appear in Journal of Economic Theory.

[2] J. Cvitanić, X. Wan, and J. Zhang, Continuous-time principal-agent problems with hidden action: the weak formulation, Working paper, University of Southern California, California, 2005.

[3] D. Duffie, P.-Y. Geoffard, and C. Skiadas, Efficient and equilibrium allocations with stochastic differential utility, Journal of Mathematical Economics 23 (1994), no. 2, 133-146.

[4] B. Dumas, R. Uppal, and T. Wang, Efficient intertemporal allocations with recursive utility, Journal of Economic Theory 93 (2000), no. 2, 240-259.

[5] B. Holmström and P. Milgrom, Aggregation and linearity in the provision of intertemporal incentives, Econometrica 55 (1987), no. 2, 303-328.

[6] Y. Hu and S. Peng, Solution offorward-backward stochastic differential equations, Probability Theory and Related Fields 103 (1995), no. 2, 273-283.

[7] K. Larsen, Optimal portfolio delegation when parties have different coefficients of risk aversion, Quantitative Finance 5 (2005), no. 5, 503-512.

[8] D. G. Luenberger, Optimization by Vector Space Methods, John Wiley \& Sons, New York, 1997.

[9] J. Ma and J. Yong, Forward-Backward Stochastic Differential Equations and Their Applications, Lecture Notes in Mathematics, vol. 1702, Springer, Berlin, 1999.

[10] H. Müller, The first-best sharing rule in the continuous-time principal agent problem with exponential utility, Journal of Economic Theory 79 (1998), 276-280.

[11] Asymptotic efficiency in dynamic principal-agent problems, Journal of Economic Theory 91 (2000), 292-301.

[12] B. Oksendal and A. Sulem, Applied Stochastic Control of Jump Diffusions, Springer, Berlin, 2004.

[13] H. Ou-Yang, Optimal contracts in a continuous-time delegated portfolio management problem, Review of Financial Studies 16 (2003), 173-208.

[14] S. Peng and Z. Wu, Fully coupled forward-backward stochastic differential equations and applications to optimal control, SIAM Journal on Control and Optimization 37 (1999), no. 3, 825-843.

[15] S. A. Ross, The economic theory of agency: the principal's problem, American Economic Review 63 (1973), 134-139.

[16] J. Yong, Completeness of security markets and solvability of linear backward stochastic dierential equations, Working paper, University of Central Florida, Florida, 2004.

[17] J. Yong and X. Y. Zhou, Stochastic Controls. Hamiltonian Systems and HJB Equations, Applications of Mathematics (New York), vol. 43, Springer, New York, 1999.

Jakša Cvitanić: Division of the Humanities and Social Sciences, Caltech, MC 228-77,

1200 E California Boulevard, Pasadena, CA 91125, USA

E-mail address: cvitanic@hss.caltech.edu

Xuhu Wan: Department of Information and Systems Management, HKUST Business School, Clear Water Bay, Kowloon, Hong Kong

E-mail address: imwan@ust.hk

Jianfeng Zhang: Department of Mathematics MC 2532, University of Southern California, 3620 S Vermont Avenue, Los Angeles, CA 90089-1113, USA

E-mail address: jianfenz@usc.edu 


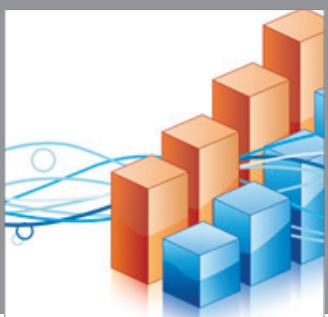

Advances in

Operations Research

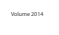

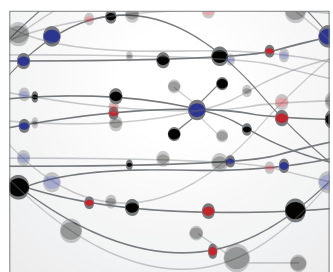

\section{The Scientific} World Journal
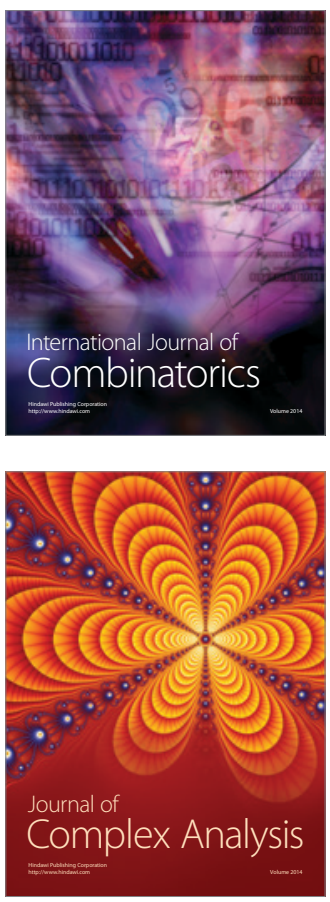

International Journal of

Mathematics and

Mathematical

Sciences
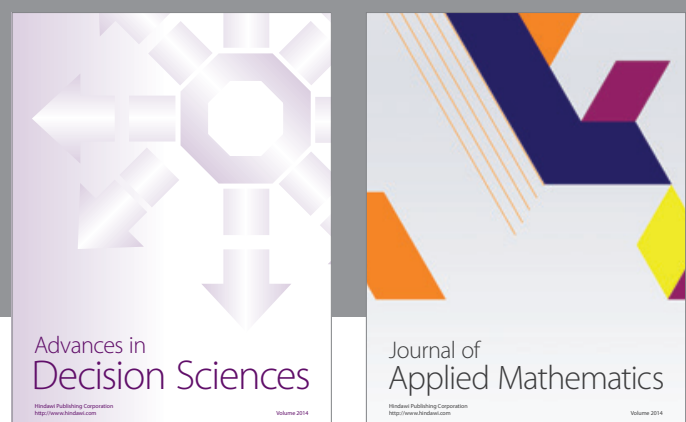

Journal of

Applied Mathematics
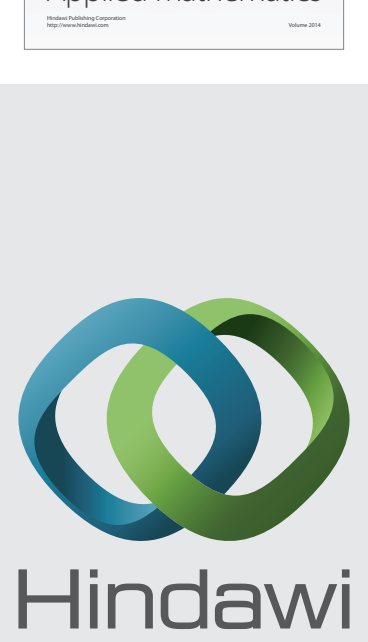

Submit your manuscripts at http://www.hindawi.com
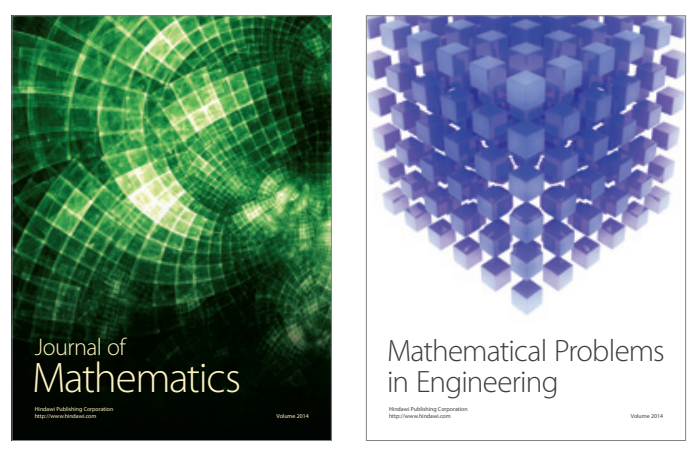

Mathematical Problems in Engineering
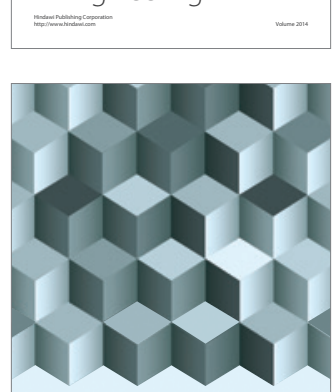

Journal of

Function Spaces


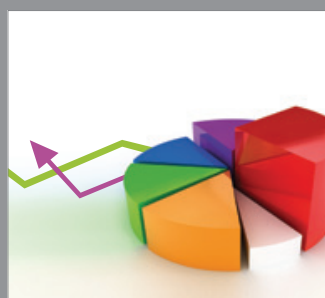

ournal of

Probability and Statistics

Promensencen
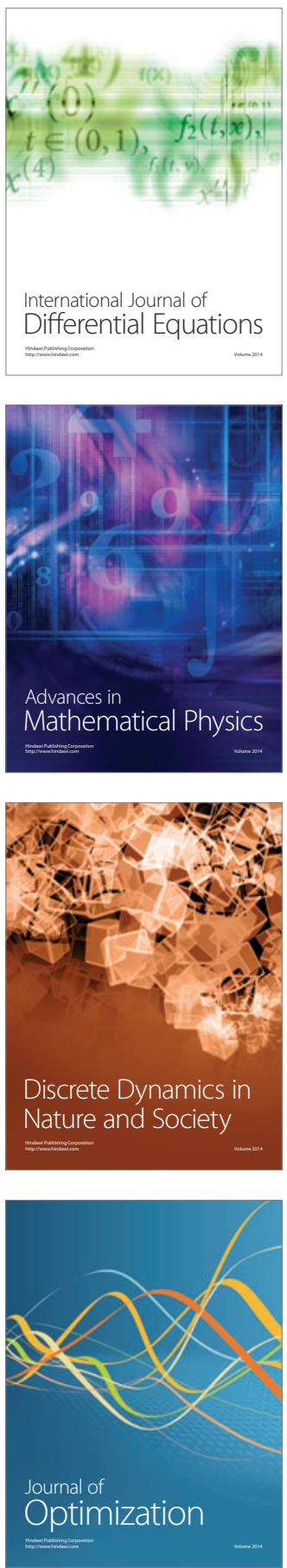